\title{
Full-sky lensing shear at second order
}

\author{
Francis Bernardeau, Camille Bonvin and Filippo Vernizzi \\ CEA, Institut de Physique Théorique, 91191 Gif-sur-Yvette cédex, France \\ CNRS, URA-2306, 91191 Gif-sur-Yvette cédex, France
}

\begin{abstract}
We compute the reduced cosmic shear up to second order in the gravitational potential without relying on the small-angle or thin-lens approximation. This is obtained by solving the Sachs equation which describes the deformation of the infinitesimal cross section of a light bundle in the optical limit, and maps galaxy intrinsic shapes into their angular images. The calculation is done in the Poisson gauge without a specific matter content, including vector and tensor perturbations generated at second order and taking account of the inhomogeneities of a fixed redshift source plane. Our final result is expressed in terms of spin-2 operators on the sphere and is valid on the full sky. Beside the well-known lens-lens and Born corrections that dominate on small angular scales, we find new nonlinear couplings. These are a purely general relativistic intrinsic contribution, a coupling between the gravitational potential at the source with the lens, couplings between the time delay with the lens and between two photon deflections, as well as nonlinear couplings due to the second-order vector and tensor components. The inhomogeneity in the redshift of the source induces a coupling between the photon redshift with the lens. All these corrections become important on large angular scales and should thus be included when computing higher-order observables such as the bispectrum, in full or partially full-sky surveys.
\end{abstract}




\section{Introduction}

In the early nineties, cosmic shear was predicted to be a promising way to study the distribution of matter in the Universe [1, 2, 3] and since its first detection [4, 5, 6, 7] it has shown to be a precious mean of investigations of the large-scale structure of the Universe, enabling us to explore dark energy properties or uncover signatures of mode coupling effects [8, 9, 10].

So far cosmic shear surveys have covered only a limited field in the sky. For instance, the CFHTLS, 1 which has produced very promising results over the last years (see e.g. [1] for a recent account of these observations), is limited to about a 170 squared degree range. With the demonstration of the robustness of cosmic shear observations, (nearly) full-sky surveys such as Pan-STARRS, DES, LSST, JDEM, or Euclid are under preparation. They will open the way to new types of studies. Akin to CMB observations, such surveys will be an excellent tool to explore the physics of the Universe at scales comparable to the Hubble radius, therefore testing genuinely general relativistic effects.2 In particular, the study of mode couplings, already well established on Newtonian scales, can be extended at these very large scales therefore testing the details of our understanding of the origin and formation of the large-scale structure.

Such an investigation requires that we know the types of mode couplings that are expected to be seen at such large scales. Calculations have been undertaken to predict the nonlinear growth of metric and density fluctuations after modes reenter the Hubble radius [13, 14, 15]. In the context of the CMB anisotropies, progress has been recently made in understanding the effect of these nonlinearities, from concentrating on the large angular scales [16, 17, 18] to the details of the physics of recombination (see for instance [19, 20]).

So far the investigations of mode couplings in weak lensing were limited to small angular scales, corresponding to scales much smaller than the angular diameter distance at the source. Accidentally, this distance roughly corresponds to the Hubble radius at the source. Thus, on these scales one can consistently neglect general relativistic effects that are suppressed by the ratio between the scale probed and the Hubble scale. On small angular scales the dominant contribution to the cosmic shear comes from fluctuations of the gravitational potential transverse to the line of sight. Perturbations along the line of sight average out and do not yield appreciable effects. In this regime the dominant geometrical mode couplings were identified more than a decade ago in [21. They include the Born correction and the lens-lens coupling. In the so-called Born approximation one integrates the lensing distortion over an unperturbed photon path. One can consider the correction due to the fact that the photon path is perturbed. The lens-lens coupling consists in the correction due to the deformation of a distant lens caused by a foreground one. The consequences of these effects have been extensively described in the literature and they have been found to have an impact on both the shear power

\footnotetext{
${ }^{1}$ http://www.cfht.hawaii.edu/Science/CFHTLS

${ }^{2}$ See 12 for a recent account of these effects on galaxy clustering observations.
} 
spectrum and higher-order statistical observables such as the bispectrum [22, 23, 24, 25, 26, 27]. As the shape distortion probes the reduced shear rather than the shear itself, there is another correction associated to the nonlinear conversion between these two quantities [28, 29, 27]. Finally, another nonlinear effect is the source-lens clustering, due to the fact that the source of the lensed light is itself a perturbed field with specific clustering properties correlated with the lens [30, 31]. For the current surveys restricted to a limited angular field all these types of couplings are undoubtedly the dominant ones.

In view of full-sky surveys one needs to go beyond the small-angle approximation and probe scales of the order of the angular diameter distance to the source. In this case fluctuations along the line of sight are not negligible and terms other than those described above may become important. Furthermore, as we are probing scales comparable to the Hubble size, one needs to undertake a full general relativistic treatment. This is important in order to compute accessible higher-order observables in full or almost full-sky surveys. In particular, in such surveys, it becomes necessary if one wants to compute the lensing bispectrum in the squeezed configuration, when one of the scales probed is taken to be much larger than the other two.

We present here the exhaustive calculation of the weak lensing cosmic shear at second order including all general relativistic contributions and without relying on the small-angle or thin-lens approximations. However, we do not include in our study the effect of source-lens clustering [30, 31 ] and other intrinsic effects in the alignment and ellipticity of galaxies. We work in the so-called generalized Poisson gauge without specifying the matter content of the Universe. We assume that there are no primordial vector and tensor perturbations. However, we will take into account vector and tensor components of the metric generated at second order from scalar fluctuations. In this gauge we derive the reduced - i.e. observable - shear by solving the Sachs equation [32] which describes the distortion of the cross section of an infinitesimal bundle of light rays in the geometric-optics limit. The advantage of using the Sachs equation instead of the geodesic equation is that it deals only with physically observable quantities. As the resolution of the Sachs equation is extremely tedious and involves a large number of terms we will develop tests that allow us to check the validity of its solution. In particular, some of the contributions to the second-order shear that we compute - and that are usually neglected in the small-angle approximation - can be compared to those expected from the lensing shear at linear order in a universe with spatial curvature.

Using the solution of the Sachs equation we will compute the reduced shear by adding the nonlinear corrections coming from the relation between this quantity and the shear itself. Finally, as in the Poisson gauge hypersurfaces of constant redshift are inhomogeneous, we take into account the corrections due to the inhomogeneity of the redshift of the source [33]. The final observable reduced shear field that we obtain is a gauge invariant quantity, although its separate contributions are not necessarily so. As it is customary for CMB polarization, we express the reduced shear in terms of spin-2 operators on the sky. In particular, angular gradients on the sky will be written in 
terms of spin raising and lowering operators, whose eigenfunctions are the well-known spin-weighted spherical harmonics.

The plan of the paper is the following. In Sec. 2 we give the outline of our calculation. In particular, we describe the Sachs equation and how the transverse size of a propagating beam can be related to the observable reduced shear including the effects of the source redshift inhomogeneities. We will solve the Sachs equation at first order in Sec. 3 while the full second-order calculation will be presented in Sec. 4. We discuss and comment on our results in Sec. 5 in the context of cosmic shear surveys regarding the generation of $B$ modes and the expected contributions to the cosmic shear bispectrum.

\section{The weak lensing equations}

We are interested in studying the propagation of a light bundle, i.e. a collection of nearby light rays [32, 34, 35, 36, 37, 38. We consider two nearby null geodesics $x^{\mu}(\lambda)$ and $x^{\mu}(\lambda)+\xi^{\mu}(\lambda)$ that lie in the past-light cone of an observer $O$, connected by a deviation vector field $\xi^{\mu}$. The affine parameter $\lambda$ is chosen in such a way that it assumes the same value at $O$ for all geodesics, i.e. $\lambda_{O}=0$. Thus, at the observer $\xi^{\mu}(0)=0$. We denote by $k^{\alpha}=\mathrm{d} x^{\alpha} / \mathrm{d} \lambda$ the wave vector of the photons. This obeys the geodesic equation,

$$
\frac{D k^{\mu}}{D \lambda}=0
$$

where $\frac{D}{D \lambda} \equiv k^{\alpha} \nabla_{\alpha}$ is the covariant derivative along the geodesic. For rays with an infinitesimal separation the connecting vector $\xi^{\mu}$ is also infinitesimal and lies on the null surface, i.e. $\xi^{\mu} k_{\mu}=0$ everywhere along the geodesics. The evolution equation of the connecting vector reads $D \xi^{\mu} / D \lambda=$ $\xi^{\nu} \nabla_{\nu} k^{\mu}$. Indeed, in some arbitrary coordinate system one has

$$
\begin{aligned}
k^{\nu} \nabla_{\nu} \xi^{\mu} & =\frac{\mathrm{d}}{\mathrm{d} \lambda} \xi^{\mu}+\Gamma_{\alpha \beta}^{\mu} k^{\alpha} \xi^{\beta} \\
& =k^{\mu}(x+\delta x)-k^{\mu}(x)+\Gamma_{\alpha \beta}^{\mu} k^{\alpha} \xi^{\beta} \\
& =\xi^{\nu} \partial_{\nu} k^{\mu}+\Gamma_{\alpha \beta}^{\mu} k^{\alpha} \xi^{\beta}
\end{aligned}
$$

By taking the covariant derivative of this equation along the photon geodesic and using the geodesic equation (1) one obtains the Sachs equation [32,

$$
\frac{D^{2} \xi^{\mu}}{D \lambda^{2}}=R_{\nu \alpha \beta}^{\mu} \xi^{\beta} k^{\nu} k^{\alpha}
$$

where we have used the Ricci identity $\left(\nabla_{\alpha} \nabla_{\beta}-\nabla_{\beta} \nabla_{\alpha}\right) k^{\mu}=R_{\nu \alpha \beta}^{\mu} k^{\nu}$ and $R_{\nu \alpha \beta}^{\mu}$ is the Riemann tensor. This equation describes the evolution of a light bundle along the geodesic.

Let us consider the case of a light beam emitted by a galaxy at spacetime position $S$ and received by an observer at $O$. We denote by $v_{O}^{\mu}$ the observer 4 -velocity. It is convenient to define an orthonormal spacelike basis $n_{a}^{\mu}$, with $a=1,2$, orthogonal to $k^{\mu}$ and to the observer velocity $v_{O}^{\mu}$ and 
such that $g_{\mu \nu} n_{a}{ }^{\nu} n_{b}{ }^{\mu}=\delta_{a b} 3$ At the observer position these vectors form a basis, $\left\{n_{1}{ }^{\mu}, n_{2}{ }^{\mu}, k^{\mu}, v_{O}^{\mu}\right\}$, which can be parallel transported along the geodesic,

$$
\frac{D n_{a}{ }^{\mu}}{D \lambda}=0, \quad \frac{D v_{O}^{\mu}}{D \lambda}=0 .
$$

The subspace defined by $\left\{n_{1}{ }^{\mu}(\lambda), n_{2}{ }^{\mu}(\lambda)\right\}$ is called the screen adapted to $v_{O}^{\mu}$ and $k^{\mu}$.

We can write the deviation vector $\xi^{\mu}(\lambda)$ in this basis. Using the fact that $\xi^{\mu} k_{\mu}=0$ along the geodesic, the component along $v_{O}^{\mu}$ vanishes and we have, for all $\lambda$,

$$
\xi^{\mu}=\xi^{a} n_{a}^{\mu}+\xi^{0} k^{\mu}
$$

with $\xi^{a}(0)=0$ and $\xi^{0}(0)=0$ at the observer. We can then plug this decomposition into the Sachs equation (3). Using the symmetry properties of the Riemann tensor the right-hand side of this equation becomes $R_{\nu \alpha \beta}^{\mu} \xi^{a} n_{a}{ }^{\beta} k^{\nu} k^{\alpha}$. Furthermore, using the equation of parallel transport for $n_{a}^{\mu}$ and $v_{O}^{\mu}$, eq. (4), and projecting the Sachs equation along the spatial basis $n_{a}^{\mu}$, one obtains an evolution equation for $\xi^{a}$,

$$
\frac{\mathrm{d}^{2} \xi^{a}}{\mathrm{~d} \lambda^{2}}=\mathcal{R}^{a}{ }_{b} \xi^{b}
$$

where the $2 \mathrm{D}$ tensor $\mathcal{R}_{a b}$ is defined by

$$
\mathcal{R}_{a b} \equiv R_{\mu \nu \rho \sigma} k^{\nu} k^{\rho} n_{a}^{\mu} n_{b}{ }^{\sigma}
$$

As eq. (6) is linear with initial condition $\xi^{a}(0)=0$, its solution can be written in the form

$$
\xi^{a}=\mathcal{D}_{a b} \theta_{O}^{b}
$$

where

$$
\left.\theta_{O}^{b} \equiv \frac{\mathrm{d} \xi^{b}}{\mathrm{~d} \lambda}\right|_{\lambda=0}
$$

is the (vectorial) angle between the photon geodesic and the neighboring one at the observer. $\mathcal{D}_{a b}$ is a linear matrix, called Jacobi mapping. It relates the angle of observation $\theta_{O}^{b}$ to the image on the screen adapted to $v_{O}^{\mu}$ and $k^{\mu}$ described by the two spatial components of $\xi^{\mu}, \xi^{a}$. From eq. (8), eq. (6) can be rewritten as an evolution equation for $\mathcal{D}_{a b}$ [35, 36, 37, 38],

$$
\frac{\mathrm{d}^{2}}{\mathrm{~d} \lambda^{2}} \mathcal{D}_{a b}=\mathcal{R}_{a c} \mathcal{D}_{c b}
$$

Equation (9) and $\xi^{a}(0)=0$ imply that $\mathcal{D}_{a b}(0)=0$ and $\mathrm{d} \mathcal{D}_{a b} /\left.\mathrm{d} \lambda\right|_{\lambda=0}=\delta_{a b}$. Note that $\mathcal{R}_{a b}$ is symmetric but $\mathcal{D}_{a b}$ is generally not.

\footnotetext{
${ }^{3}$ More generally, it is possible to introduce an induced 2D metric on the subspace described by $n_{a}{ }^{\mu}$ by imposing $g_{\mu \nu} n_{a}{ }^{\nu} n_{b}{ }^{\mu}={ }^{2} \mathrm{D}_{a b}$. This is particularly convenient when employing spherical polar coordinates to describe the $3 \mathrm{D}$ space. In this case the Latin indices $a, b, \ldots$ are raised and lowered by the metric ${ }^{2 \mathrm{D}} g_{a b}$. As here we take ${ }^{2 \mathrm{D}} g_{a b}=\delta_{a b}$, upper or lower positions of the indices are irrelevant and repeated indices represent a summation over $a=1,2$.
} 
We can decompose the linear mapping $\mathcal{D}_{a b}$ into a spin-0 component and a spin-2 component, respectively,

$$
\begin{aligned}
& { }_{0} \mathcal{D} \equiv \mathcal{D}_{11}+\mathcal{D}_{22}+\mathrm{i}\left(\mathcal{D}_{12}-\mathcal{D}_{21}\right) \\
& { }_{2} \mathcal{D} \equiv \mathcal{D}_{11}-\mathcal{D}_{22}+\mathrm{i}\left(\mathcal{D}_{12}+\mathcal{D}_{21}\right)
\end{aligned}
$$

The spin- 2 part, ${ }_{2} \mathcal{D}$, is directly related to the usual shear spin-2 field $\gamma \equiv \gamma_{1}+\mathrm{i} \gamma_{2}$. Indeed, by defining the vectorial angular position at the source as $\theta_{S}^{a} \equiv \xi^{a} / \lambda_{S}$ one finds the following relations 38

$$
\gamma_{1}=-\frac{1}{2 \lambda_{S}}\left(\mathcal{D}_{11}-\mathcal{D}_{22}\right), \quad \gamma_{2}=-\frac{1}{2 \lambda_{S}}\left(\mathcal{D}_{12}+\mathcal{D}_{21}\right) .
$$

The spin-0 part, ${ }_{0} \mathcal{D}$, contains a real part, the trace $\mathcal{D}=\mathcal{D}_{11}+\mathcal{D}_{22}$, which is related to the usual convergence by

$$
\kappa=1-\frac{1}{2 \lambda_{S}} \mathcal{D}
$$

The imaginary part comes from the fact that, unlike $\mathcal{R}_{a b}, \mathcal{D}_{a b}$ is not necessarily symmetric. Indeed, as we will see, it is not symmetric at second order. The imaginary part of ${ }_{0} \mathcal{D}$ corresponds to a rotation of the observed object and it is related to the usual rotation parameter $\omega$ by

$$
\omega=-\frac{1}{2 \lambda_{S}}\left(\mathcal{D}_{12}-\mathcal{D}_{21}\right) \text {. }
$$

At second order, the rotation has no observational consequences on the observed galaxy polarization 4 Therefore we will ignore it in the following. We are thus left with one scalar degree of freedom describing the convergence and 2 degrees of freedom for the shear. The latter can be mapped into the so-called "electric" and "magnetic" modes (see Appendix B). Note that at second order the electric mode is not necessarily equal to the convergence field as in the linear case.

What we observe is the ratio between the anisotropic and isotropic deformations, i.e. the reduced shear, defined as 39]

$$
g \equiv \frac{\gamma}{1-\kappa} .
$$

From eqs. (13) and (14) this is given, in terms of the Jacobi mapping $\mathcal{D}_{a b}$, by

$$
g=-{ }_{2} \mathcal{D} / \mathcal{D}
$$

In the following we will solve eq. (10) in a perturbed universe. For the background we will assume a flat Friedmann-Lemaître-Robertson-Walker (FLRW) metric given by $\mathrm{d} s^{2}=a^{2}(\eta)\left(-\mathrm{d} \eta^{2}+\mathrm{d} \mathbf{x}^{2}\right)$,

\footnotetext{
${ }^{4}$ More precisely, one can define the observed complex shape polarization as $p=\left(m_{11}-m_{22}+2 \mathrm{i} m_{12}\right) /\left(m_{11}+\right.$ $\left.m_{22}\right)$ where $m_{i j}$ is the luminosity distribution matrix of observed galaxies. For an unpolarized source and in absence of rotation this is $p=2 g /(1+g g *)$ [39]. In case of rotation this relation becomes $\left(1-\tilde{\kappa}^{*}\right) /(1-\tilde{\kappa}) p=$ $2 \tilde{g} /\left(1+\tilde{g} \tilde{g}^{*}\right)$ where $\tilde{\kappa}$ is complex and defined as $\tilde{\kappa} \equiv \kappa+\mathrm{i} \omega$ and $\tilde{g} \equiv g /(1-\tilde{\kappa})$. Thus, as $\tilde{\kappa}$ is real at linear order and $\gamma$ vanishing at zeroth order, the imaginary part of $\tilde{\kappa}$ enters in the expression of the observed polarization at third order only.
} 
where $\eta$ is the conformal time. Since null geodesics are not affected by conformal transformations, it will be convenient to perform the calculation without the Friedmann expansion and reintroduce the effect of the expansion only at the end. Indeed, as shown in Appendix $\mathrm{A}$, the effect of the expansion can be simply taken into account by rescaling the mapping $\mathcal{D}_{a b}$ by the scale factor, e.g. $\mathcal{D}_{a b} \rightarrow a \mathcal{D}_{a b}$. Note that, as both ${ }_{2} \mathcal{D}$ and $\mathcal{D}$ get rescaled by the conformal transformation, the reduced shear (17) is not affected by the expansion. We parametrize the photon geodesic such that $x^{0}(\lambda(\eta))=\eta_{0}-\eta$. It is thus convenient to define $\chi \equiv \eta_{0}-\eta$ so that

$$
k^{0}=\frac{\mathrm{d} \chi}{\mathrm{d} \lambda}
$$

and the evolution equation (10) now reads

$$
\frac{\mathrm{d}^{2}}{\mathrm{~d} \chi^{2}} \mathcal{D}_{a b}+\frac{1}{k^{0}} \frac{\mathrm{d} k^{0}}{\mathrm{~d} \chi} \frac{\mathrm{d}}{\mathrm{d} \chi} \mathcal{D}_{a b}=\frac{1}{\left(k^{0}\right)^{2}} \mathcal{R}_{a c} \mathcal{D}_{c b}
$$

We can solve this second-order differential equation order by order in the metric perturbations.

The solution of eq. (19) gives the linear mapping between the observed angle and the shape of a source at a given coordinate time $\eta_{S}$. Observationally, we are interested in a mapping where the source is defined at a given redshift $z_{S}$. As in an inhomogeneous universe the redshift is a perturbed quantity, at second order we expect a contribution to the reduced shear due to the coupling between this perturbation and the lenses. Thus, the reduced shear at constant redshift is given by

$$
g_{z}=g-\frac{\mathrm{d} g}{\mathrm{~d} z_{S}} \delta z_{S},
$$

where $\delta z=z+1-a_{0} / a$ is the perturbation of the redshift. As this correction is not conformally invariant, it introduces a dependence on the expansion. At first order, though, only the trace of the linear mapping, $\mathcal{D}$, is affected by redshift perturbations [33], but the traceless part is not and therefore in this case $g_{z}=g$.

\section{The shear and the convergence at first order}

As a warm up exercise, before the calculation of the shear at second order we derive here, using eq. (19), the shear and the convergence at first order. We consider a perturbed FLRW metric in Newtonian gauge, written in Cartesian coordinates as

$$
\mathrm{d} s^{2}=a^{2}(\eta)\left[-(1+2 \phi) \mathrm{d} \eta^{2}+(1-2 \psi) \mathrm{d} \mathbf{x}^{2}\right],
$$

where we have neglected primordial vector and tensor perturbations. For convenience, we define also the Weyl potential $\Psi$ as

$$
\Psi \equiv(\phi+\psi) / 2
$$


and we will use it in the following whenever the combination $\phi+\psi$ appears. As explained in the previous section, the reduced shear (17) is not affected by the expansion and we can set the scale factor $a=1$.

Let us define $k^{\mu}(0) \equiv\left(1, \mathbf{e}_{r}\right)$ as the photon 4-momentum at the observer, where $\mathbf{e}_{r}$ defines the direction of the line of sight. Note that in this definition we have set the metric perturbations at the observer position to zero. The final result will be independent of this choice. Indeed, since metric perturbations at the observer do not depend on the direction of observation, they can be reabsorbed into the homogeneous mapping. As in flat spacetime the Christoffell symbols vanish, $k^{\mu}(0)$ is parallel propagated along the background geodesic, while the curvature tensor $R_{\mu \alpha \beta \nu}$ vanishes thus making $\mathcal{R}_{a b}$ at least a first-order quantity. Then, at first order in perturbations eq. (19) simplifies to

$$
\frac{\mathrm{d}^{2}}{\mathrm{~d} \chi^{2}} \mathcal{D}_{a b}+\frac{\mathrm{d} k^{0}}{\mathrm{~d} \chi} \frac{\mathrm{d}}{\mathrm{d} \chi} \mathcal{D}_{a b}=\mathcal{R}_{a c} \mathcal{D}_{c b}
$$

where we have used that $\mathrm{d} k^{0} / \mathrm{d} \chi$ vanishes on the background.

On the background this equation becomes $\mathrm{d}^{2} \mathcal{D}_{a b} / \mathrm{d} \chi^{2}=0$. Requiring that the homogeneous mapping is proportional to the unit matrix yields $\mathcal{D}_{a b}=\chi \delta_{a b}$ for the background solution. Furthermore, we can plug the background solution for $\mathcal{D}_{a b}$ in the second term on the left-hand side and on the right-hand side of eq. (23) to obtain

$$
\frac{\mathrm{d}^{2}}{\mathrm{~d} \chi^{2}} \mathcal{D}_{a b}+\frac{\mathrm{d} k^{0}}{\mathrm{~d} \chi} \delta_{a b}=\chi \mathcal{R}_{a b}
$$

We then integrate this equation once up to the source using the boundary condition $\mathrm{d} \mathcal{D}_{a b} /\left.\mathrm{d} \chi\right|_{O}=\delta_{a b}$,

$$
\frac{\mathrm{d}}{\mathrm{d} \chi} \mathcal{D}_{a b}=\left(2-k^{0}\right) \delta_{a b}+\int_{0}^{\chi} \mathrm{d} \chi^{\prime} \chi^{\prime} \mathcal{R}_{a b}
$$

The solution of this equation, after an integration by parts, can be written as

$$
\mathcal{D}_{a b}\left(\chi_{S}\right)=\int_{0}^{\chi_{S}} \mathrm{~d} \chi\left(2-k^{0}\right) \delta_{a b}+\int_{0}^{\chi_{S}} \mathrm{~d} \chi\left(\chi_{S}-\chi\right) \chi \mathcal{R}_{a b}
$$

$k^{0}$ can be obtained by solving the photon geodesic (1) at first order,

$$
k^{0}=1-2 \phi+\int_{0}^{\chi} d \chi^{\prime} 2 \dot{\Psi}
$$

where the dot denotes a partial derivative with respect to $\chi$, i.e. $\equiv \partial / \partial \chi$.

Let us now compute $\mathcal{R}_{a b}$. We denote by $n_{a}{ }^{\mu}(0) \equiv\left(0, \mathbf{e}_{a}\right)$ the spatial basis at the observer. As for the 4-momentum of the photon, $n_{a}^{\mu}(0)$ is parallel propagated along the background geodesic. Thus, according to the definition (7), in order to compute $\mathcal{R}_{a b}$ we need to contract the curvature tensor at first order with the unperturbed $k^{\mu}(0)$ and $n_{a}{ }^{\mu}(0)$, which yields

$$
\mathcal{R}_{a b}=-e_{a}^{i} e_{b}^{j} 2 \Psi_{, i j}-\frac{\mathrm{d}^{2} \psi}{\mathrm{d} \chi^{2}} \delta_{a b}
$$


Plugging $\mathcal{R}_{a b}$ given in this equation and the expression for $k^{0}$ given in eq. (27) into eq. (26) and integrating by parts $\mathrm{d}^{2} \psi / \mathrm{d} \chi^{2}$ we obtain the Jacobi mapping at linear order,

$$
\mathcal{D}_{a b}\left(\chi_{S}\right)=\left(1-\psi\left(\chi_{S}\right)\right) \chi_{S} \delta_{a b}+\int_{0}^{\chi_{S}} \mathrm{~d} \chi\left[4 \Psi-2\left(\chi_{S}-\chi\right) \dot{\Psi}\right] \delta_{a b}-e_{a}^{i} e_{b}^{j} \int_{0}^{\chi_{S}} \mathrm{~d} \chi\left(\chi_{S}-\chi\right) \chi 2 \Psi_{, i j}
$$

According to the Born approximation we can evaluate the integral along the background geodesic so that in this expression $\Psi=\Psi\left(\chi, \mathbf{e}_{r} \chi\right)$.

Note that the metric (21) is conformal to $\mathrm{d} s^{2}=-(1+4 \Psi) \mathrm{d} \eta^{2}+\mathrm{d} \mathbf{x}^{2}$. Thus, as photon geodesics are conformally invariant, one would naively expect $\mathcal{D}_{a b}$ to depend only on the combination $\Psi=(\phi+\psi) / 2$ [37. This is too quick a conclusion. Indeed, parallel transport of the basis $n_{a}{ }^{\mu}$ is not conformally invariant and the basis is deformed by the spatial curvature at the source position. For this reason the first term of eq. (29) also depends on the curvature potential at the source, $\psi_{S}$.

We now want to extract the shear and convergence from the mapping $\mathcal{D}_{a b}\left(\chi_{S}\right)$. In order to do so, it is useful to introduce spin operators on the sphere (see Appendix (B)). To each point of a 2D Riemannian manifold described by an orthonormal basis $\left\{\mathbf{e}_{1}, \mathbf{e}_{2}\right\}$ we can associate a spin- $s$ field ${ }_{s} X$ such that under the rotation of $\mathbf{e}_{a}$ by an angle $\alpha$ it transforms as ${ }_{s} X \rightarrow e^{\mathrm{i} \alpha{ }_{s}} X$ (for more details see [40, 41, 42]). More precisely, following [41], the local freedom in the choice of the basis is equivalent to the transformations

$$
\mathbf{e}_{ \pm} \equiv \mathbf{e}_{1} \pm \mathrm{i} \mathbf{e}_{2} \rightarrow e^{\mathrm{i} \alpha} \mathbf{e}_{ \pm}
$$

To every spin- $s{ }_{s} X$ we can associate a symmetric and trace-free tensor of rank $s \geq 0, X_{a_{1} \ldots a_{s}}$ : for $s \geq 0$,

$$
X^{a_{1} \ldots a_{s}} \equiv 2^{-s}{ }_{s} X e_{-}^{a_{1}} \cdots e_{-}^{a_{s}}
$$

The inverse relation is

$$
{ }_{s} X \equiv e_{+}^{a_{1}} \cdots e_{+}^{a_{s}} X_{a_{1} \ldots a_{s}} .
$$

For $s<0$ we define $X^{a_{1} \cdots a_{|s|}} \equiv 2^{-|s|} X e_{+}^{a_{1}} \cdots e_{+}^{a_{|s|}}$.

As we are interested in describing the lensing field on the sphere of the sky, for the orthonormal basis we choose the two coordinate basis vectors of a spherical polar coordinate system, $\left\{\mathbf{e}_{1}, \mathbf{e}_{2}\right\} \equiv$ $\left\{\mathbf{e}_{\theta}, \mathbf{e}_{\varphi}\right\}$. Let us rewrite the three spatial vectors $\mathbf{e}_{r}$ and $\mathbf{e}_{a}$ in the Cartesian representation as

$$
\begin{aligned}
\mathbf{e}_{r} & =(\sin \theta \cos \varphi, \sin \theta \sin \varphi, \cos \theta) \\
\mathbf{e}_{\theta} & =(\cos \theta \cos \varphi, \cos \theta \sin \varphi,-\sin \theta) \\
\mathbf{e}_{\varphi} & =(-\sin \varphi, \cos \varphi, 0)
\end{aligned}
$$

where $\theta, \varphi$ are the angles of observation. Furthermore, we can define operators that increase or decrease the index of the spin by 1 ,

$$
\not \partial{ }_{s} X \equiv-\sin ^{s} \theta\left(\partial_{\theta}+\mathrm{i} \csc \theta \partial_{\varphi}\right)\left(\sin ^{-s} \theta\right){ }_{s} X, \quad \bar{\partial}_{s} X \equiv-\sin ^{-s} \theta\left(\partial_{\theta}-\mathrm{i} \csc \theta \partial_{\varphi}\right)\left(\sin ^{s} \theta\right){ }_{s} X .
$$


With the definitions (33)-(35) above we have

$$
e_{r}^{i} \partial_{i}=\partial_{r}, \quad e_{\theta}^{i} \partial_{i}=\frac{1}{\chi} \partial_{\theta}, \quad e_{\varphi}^{i} \partial_{i}=\frac{1}{\chi \sin \theta} \partial_{\varphi},
$$

where we have used that $r=\chi$ along the photon geodesic. Using these relations it is easy to verify that, if $X=X(\chi)$ is a scalar field,

$$
e_{+}^{i} X_{, i}=-\frac{1}{\chi} \not \partial X, \quad e_{-}^{i} X_{, i}=-\frac{1}{\chi} \bar{\partial} X .
$$

Then, employing the useful relation $\chi e_{ \pm}^{i} \partial_{i} e_{ \pm}^{j}=\cot \theta e_{ \pm}^{j}$ one can verify that

$$
e_{+}^{i} e_{+}^{j} X_{, i j}=\frac{1}{\chi^{2}} \not \not^{2} X, \quad e_{-}^{i} e_{-}^{j} X_{, i j}=\frac{1}{\chi^{2}} \bar{\not}^{2} X,
$$

and, analogously with $\chi e_{\mp}^{i} \partial_{i} e_{ \pm}^{j}=-\cot \theta e_{ \pm}^{j}-2 e_{r}^{j}$, that

$$
e_{+}^{i} e_{-}^{j} X_{, i j}=e_{-}^{i} e_{+}^{j} X_{, i j}=\frac{1}{\chi^{2}} \bar{\partial} \not \partial X-\frac{2}{\chi} X_{, r} .
$$

Note that the 2D Laplacian on the sphere is given by $\not \supset \bar{\partial} X=\bar{\not} \not \partial X$.

Let us first apply these definitions to $\mathcal{R}_{a b}$. By making use of eq. (32) we can define a spin-2 field on the sphere, ${ }_{2} \mathcal{R} \equiv e_{+}^{a} e_{+}^{b} \mathcal{R}_{a b}$, which by eq. (39) reads

$$
{ }_{2} \mathcal{R}=-\frac{2}{\chi^{2}} \not \partial^{2} \Psi
$$

Then, using that $e_{a}^{i} e_{a}^{j}=\left(e_{+}^{i} e_{-}^{j}+e_{-}^{i} e_{+}^{j}\right) / 2$ and eq. (40), the trace of $\mathcal{R}_{a b}$ reads

$$
\mathcal{R}=-\frac{4}{\chi} \Psi_{, r}-\frac{2}{\chi^{2}} \bar{\not} \not \partial \Psi-2 \frac{\mathrm{d}^{2} \psi}{\mathrm{d} \chi^{2}} .
$$

We can do the same for the mapping (29). One finds the spin-2 mapping field,

$$
{ }_{2} \mathcal{D}\left(\chi_{S}\right)=-2 \int_{0}^{\chi_{S}} \mathrm{~d} \chi \frac{\chi_{S}-\chi}{\chi} \not \not^{2} \Psi
$$

At first order the reduced shear $g$ is given by ${ }_{2} \mathcal{D}$ divided by the background part of $\mathcal{D}$, which is simply $\overline{\mathcal{D}}=2 \chi_{S}$, i.e.

$$
g=\int_{0}^{\chi_{S}} \mathrm{~d} \chi \frac{\chi_{S}-\chi}{\chi \chi_{S}} \not \not^{2} \Psi
$$

Taking the trace of eq. (29) and defining $\delta \mathcal{D}=\mathcal{D}-2 \chi$ one finds, after an integration by part,

$$
\delta \mathcal{D}\left(\chi_{S}\right)=-2 \psi\left(\chi_{S}\right) \chi_{S}+2 \int_{0}^{\chi_{S}} \mathrm{~d} \chi\left(2 \Psi-\frac{\chi_{S}-\chi}{\chi} \not \partial \not \partial\right)
$$

which is proportional to the convergence, $\kappa$. The first two terms on the right-hand side are not usually included in the convergence because they are negligible on small angular scales 5 In particular, the

\footnotetext{
${ }^{5}$ The Laplacian $\bar{\not} \not \partial$ introduces a factor $l(l+1)$ in harmonic space, thus enhancing the contribution to the power spectrum from the third term of eq. (45) with respect to the contribution of the other terms.
} 
first term is a relativistic effect due to the deformation of the size of the source caused by the curvature potential at the source. The second term is the Shapiro time delay [43]. Both effects are negligible on small angular scales.

We can check that the first two terms in eq. (45) contribute to the convergence by considering a nonexpanding FLRW universe with nonzero constant spatial curvature $K$ and metric

$$
d s^{2}=-\mathrm{d} \eta^{2}+\frac{1}{\left(1+\psi_{K}(r)\right)^{2}}\left(d r^{2}+r^{2} d \Omega^{2}\right) \quad \text { with } \quad \psi_{K}(r) \equiv \frac{K r^{2}}{4} .
$$

In this universe the angular diameter distance is

$$
D_{A}(\chi)= \begin{cases}\sin (\sqrt{K} \chi) / \sqrt{K} & \text { for } \quad K>0, \\ \sinh (\sqrt{|K|} \chi) / \sqrt{|K|} & \text { for } \quad K<0 .\end{cases}
$$

For small curvature, $K \ll 1$, the expression above can be expanded at first order in $K$ to give

$$
D_{A}\left(\chi_{S}\right)=\chi_{S}-\frac{K \chi_{S}^{3}}{6}
$$

The first term on the right-hand side is the angular diameter distance in a spatially flat universe while the second term is a small perturbation to it, due to the curvature.

As the trace $\mathcal{D}$ corresponds to twice the angular diameter distance, we can check that $\delta \mathcal{D}\left(\chi_{S}\right) / 2$, with $\delta \mathcal{D}\left(\chi_{S}\right)$ given in eq. (45), correctly reproduces this small perturbation in this particular case. For $\phi=0$ and $\psi=\psi_{K}$ (and $a=1$ ) the flat perturbed metric in Poisson gauge, eq. (21), reproduces at first order in $\psi_{K}$ the spatially curved metric (46). Thus, in this case $\Psi \equiv(\phi+\psi) / 2=K r^{2} / 8$. As the Weyl potential $\Psi$ depends only on the radial coordinate, the last term on the right-hand side of eq. (45) vanishes so that it does not contribute to the angular diameter distance. Furthermore, the contributions from the first and the second term of eq. (45) are, respectively, $-K \chi_{S}^{3} / 4$ and $K \chi_{S}^{3} / 12$, where we have used that, at lowest order in $\psi_{K}, r=\chi$ along the photon geodesic. This yields $\delta \mathcal{D}\left(\chi_{S}\right) / 2=-K \chi_{S}^{3} / 6$, i.e. the second term of eq. (48). Note that the first two terms of eq. (45) have no counterpart in the shear. Indeed, a constant curvature deviates light rays only isotropically. Furthermore, Kaiser's relation [44] between shear and convergence, $\not \partial \kappa=\bar{\partial} \gamma$, is only valid in the limit of small angles, when the first two terms of eq. (45) are negligible.

\section{The shear at second order}

In this section we will compute the lensing shear at second order. As we concentrate on the shear, we will compute only the trace-free and symmetric part of the matrix $\mathcal{D}_{a b}$. Indeed, at second order the trace and the antisymmetric part contribute only to the convergence $\kappa$ and the rotation $\omega$, respectively. We will do the calculation in the so-called generalized Poisson gauge, where the second-order metric can be written as [45]

$$
\mathrm{d} s^{2}=a^{2}(\eta)\left[-e^{2 \phi} \mathrm{d} \eta^{2}+2 \omega_{i} \mathrm{~d} \eta \mathrm{d} x^{i}+\left(e^{-2 \psi} \delta_{i j}+h_{i j}\right) \mathrm{d} x^{i} \mathrm{~d} x^{j}\right] .
$$


Here the vector component $\omega_{i}$ is divergenceless, $\partial_{i} \omega_{i}=0$, and the tensor component $h_{i j}$ is divergenceless and traceless, $\partial_{i} h_{i j}=0=h_{i i}$. As we neglect primordial vector and tensor perturbations, $\omega_{i}$ and $h_{i j}$ are only second-order quantities. Note that we have used the exponential form for the gravitational potentials in the metric. Indeed, in this form the metric is conformal to $\mathrm{d} s^{2}=-e^{4 \Psi} \mathrm{d} \eta^{2}+2 \omega_{i} \mathrm{~d} \eta \mathrm{d} x^{i}+\left(\delta_{i j}+h_{i j}\right) \mathrm{d} x^{i} \mathrm{~d} x^{j}$ so that the effect of scalar perturbations on the null geodesic is only through the Weyl potential $\Psi=(\phi+\psi) / 2$. As done in the previous section, we will drop the effect of the expansion setting $a=1$. We will reintroduce the expansion in Sec. 4.3.

\subsection{Solving the Sachs equation}

To solve the Sachs equation at second order let us go back to eq. (19). This can be integrated to obtain

$$
\frac{\mathrm{d}}{\mathrm{d} \chi} \mathcal{D}_{a b}=\delta_{a b}+\int_{0}^{\chi} \mathrm{d} \chi^{\prime}\left(\frac{1}{\left(k^{0}\right)^{2}} \mathcal{R}_{a c} \mathcal{D}_{c b}-\frac{1}{k^{0}} \frac{\mathrm{d} k^{0}}{\mathrm{~d} \chi} \frac{\mathrm{d}}{\mathrm{d} \chi} \mathcal{D}_{a b}\right)
$$

where we have used that $\mathrm{d} \mathcal{D}_{a b} /\left.\mathrm{d} \chi\right|_{O}=\delta_{a b}$ at the observer. Since $\mathrm{d} k^{0} / \mathrm{d} \chi$ vanishes on the background we can rewrite the last term of this equation using the first-order equation (25) and integrate eq. (50) up to the source $S$. We obtain, after integration by parts,

$$
\mathcal{D}_{a b}\left(\chi_{S}\right)=\chi_{S} \delta_{a b}+\int_{0}^{\chi_{S}} \mathrm{~d} \chi \frac{\chi_{S}-\chi}{\chi} \mathcal{S}_{a b}
$$

where we have defined the source term $\mathcal{S}_{a b}$ as

$$
\mathcal{S}_{a b} \equiv \frac{\chi}{\left(k^{0}\right)^{2}} \mathcal{R}_{a c} \mathcal{D}_{c b}-\frac{\chi}{k^{0}} \frac{\mathrm{d} k^{0}}{\mathrm{~d} \chi}\left(2-k^{0}\right) \delta_{a b}-\chi \frac{\mathrm{d} k^{0}}{\mathrm{~d} \chi} \int_{0}^{\chi} \mathrm{d} \chi^{\prime} \chi^{\prime} \mathcal{R}_{a b}
$$

At second order we need to go beyond the standard Born approximation. Since the source term $\mathcal{S}_{a b}$ is at least first order, we just need to integrate along a geodesic which is perturbed at first order. Thus, we evaluate the source term at the perturbed geodesic position $x_{\text {pert }}^{i}(\chi)=x^{i}(\chi)+\delta x^{i}(\chi)$, where $\delta x^{i}$ is the geodesic deviation. Expanding $\mathcal{S}_{a b}$ along the background geodesic,

$$
\mathcal{S}_{a b}\left(x_{\text {pert }}^{i}\right)=\mathcal{S}_{a b}\left(x^{i}\right)+\left.\delta x^{j} \cdot \delta\left(\mathcal{S}_{a b}\right)_{j}\right|_{x}
$$

where the shift $\delta\left(\mathcal{S}_{a b}\right)_{j}$ will be computed below, we can rewrite the source term $\mathcal{S}_{a b}$ as a function of the unperturbed geodesic position $x^{i}$ as

$$
\mathcal{S}_{a b} \equiv \frac{\chi^{2}}{\left(k^{0}\right)^{2}} \mathcal{R}_{a b}+\chi \mathcal{R}_{a c} \delta \mathcal{D}_{c b}-\frac{\chi}{k^{0}} \frac{\mathrm{d} k^{0}}{\mathrm{~d} \chi}\left(2-k^{0}\right) \delta_{a b}-\chi \frac{\mathrm{d} k^{0}}{\mathrm{~d} \chi} \int_{0}^{\chi} \mathrm{d} \chi^{\prime} \chi^{\prime} \mathcal{R}_{a b}+\chi^{2} \delta x^{j} \cdot \delta\left(\mathcal{R}_{a b}\right)_{j},
$$

where to write the first two terms we have separated $\mathcal{D}_{a b}$ into its background and first-order part as $\mathcal{D}_{a b}=\chi \delta_{a b}+\delta \mathcal{D}_{a b}$ and we have employed for the last term that at leading order $\mathcal{S}_{a b}=\chi^{2} \mathcal{R}_{a b}$. We will now compute the symmetric traceless part of the source $\mathcal{S}_{a b}$ evaluating one by one each of the terms on the right-hand side of eq. (54).

Let us start with the first term in eq. (54). Since on a flat background spacetime the unperturbed part of $R_{\mu \alpha \beta \nu}$ vanishes, according to the definition (7), i.e., $\mathcal{R}_{a b}=R_{\mu \alpha \beta \nu} k^{\alpha} k^{\beta} n_{a}{ }^{\mu} n_{b}{ }^{\nu}$, in order to 
compute $\mathcal{R}_{a b}$ up to second order we just need to consider the photon wave vector $k^{\mu}$ and the basis $n_{a}^{\mu}$ up to first order. Integrating the geodesic equation (11) one obtains, up to first order,

$$
\begin{aligned}
k^{0} & =1-2 \phi+\int_{0}^{\chi} d \chi^{\prime} 2 \dot{\Psi}, \\
k^{i} & =(1+2 \psi) e_{r}^{i}-\int_{0}^{\chi} d \chi^{\prime} 2 \partial^{i} \Psi .
\end{aligned}
$$

Integrating up to first order the parallel transport equation for $n_{a}^{\mu}$, eq. (4), one finds

$$
\begin{aligned}
& n_{a}{ }^{0}=-e_{a}^{i} \int_{0}^{\chi} d \chi^{\prime} \partial_{i} \phi, \\
& n_{a}^{i}=e_{a}^{i} \psi+e_{r}^{i} e_{a}^{j} \int_{0}^{\chi} d \chi^{\prime} \partial_{j} \psi .
\end{aligned}
$$

Since the vector and tensor parts of the metric are already second order, they enter linearly in $R_{\mu \alpha \beta \nu}$, which just needs to be contracted with the unperturbed $k^{\mu}$ and $n_{a}^{\mu}$. Furthermore, it is convenient to compute the ratio $k^{i} / k^{0}$ which appears when combining $k^{i}$ in the definition (7) with the denominator of the first term of eq. (54). This yields

$$
\frac{k^{i}}{k^{0}}=e_{r}^{i}(1+2 \Psi)-e_{a}^{i} e_{a}^{j} \int_{0}^{\chi} \mathrm{d} \chi^{\prime} 2 \Psi_{, j}
$$

Finally, computing $R_{\mu \alpha \beta \nu}$ up to second order and making use of the definition (7), the traceless part of the first term of eq. (54) reads

$$
\begin{aligned}
\frac{\chi^{2}}{\left(k^{0}\right)^{2}}\left(\mathcal{R}_{a b}\right)^{T}= & -\chi^{2} e_{(a}^{i} e_{b)}^{j}\left(2 \Psi_{, i j}+4 \Psi_{, i} \Psi_{, j}+8 \Psi \Psi_{, i j}+8 e_{r}^{k} \Psi_{, i k} \int_{0}^{\chi} \Psi_{, j}\right. \\
& \left.-e_{r}^{k} \omega_{k, i j}+\frac{\mathrm{d}}{\mathrm{d} \chi} \omega_{i, j}-\frac{1}{2} e_{r}{ }^{k} e_{r}^{l} h_{k l, i j}-\frac{1}{2} \frac{\mathrm{d}^{2}}{\mathrm{~d} \chi^{2}} h_{i j}+\frac{1}{2} \frac{\mathrm{d}}{\mathrm{d} \chi} e_{r}{ }^{k} h_{k i, j}\right),
\end{aligned}
$$

where the parenthesis in the indices denote symmetrization. After few manipulations, employing eqs. (38) and (39), and the useful relation $\chi e_{+}^{i} \partial_{i} e_{r}^{j}=e_{+}^{j}$, we can write its contribution to ${ }_{2} \mathcal{S} \equiv$ $e_{+}^{a} e_{+}^{b} \mathcal{S}_{a b}$ as

$$
\begin{aligned}
& -2 \not \partial^{2} \Psi-4 \not \partial \Psi \not \partial \Psi-8 \Psi \not \partial^{2} \Psi-8\left(\chi \not \partial \Psi_{, r}-\not \partial \Psi\right) \int_{0}^{\chi} \mathrm{d} \chi^{\prime} \frac{1}{\chi^{\prime}} \not \partial \Psi \\
& +\not \partial^{2} \omega_{r}+\frac{\mathrm{d}}{\mathrm{d} \chi}\left(\chi \not \partial{ }_{1} \omega\right)+\frac{1}{2} \not \partial^{2} h_{r r}+\frac{\mathrm{d}}{\mathrm{d} \chi}\left(\chi \not \partial_{1} h_{r}\right)+\frac{\chi}{2} \frac{\mathrm{d}^{2}}{\mathrm{~d} \chi^{2}}\left(\chi_{2} h\right),
\end{aligned}
$$

where $\omega_{r} \equiv e_{r}^{i} \omega_{i}, h_{r r} \equiv e_{r}^{i} e_{r}^{j} h_{i j}$ and we have defined the spin-1 part of $\omega,{ }_{1} \omega \equiv e_{+}{ }^{i} \omega_{i}$, the spin-1 quantity ${ }_{1} h_{r} \equiv e_{+}^{i} e_{r}^{j} h_{i j}$ and the spin-2 part of the tensor mode $h_{i j},{ }_{2} h \equiv e_{+}{ }^{i} e_{+}^{j} h_{i j} 6$

\footnotetext{
${ }^{6}$ Note that $\omega_{i}$ and $h_{i j}$ can be decomposed respectively as $\omega_{i}=\omega_{r} e_{r}^{i}+\frac{1}{2}-1 \omega e_{+}^{i}+\frac{1}{2} 1 \omega e_{-}^{i}$ and $h_{i j}=$ $h_{r r}\left(e_{r}^{i} e_{r}^{j}-\frac{1}{2} e_{+}^{(i} e_{-}^{j)}\right)+{ }_{-1} h_{r} e_{+}^{(i} e_{r}^{j)}+{ }_{1} h_{r} e_{-}^{(i} e_{r}^{j)}+\frac{1}{4}-2 h e_{+}^{i} e_{+}^{j}+\frac{1}{4} 2 h e_{-}^{i} e_{-}^{j}$. These components are not independent, as they are related by the divergenceless conditions $\omega_{i, i}=0=h_{i j, i}$.
} 
The second term of eq. (54) is the product of two first-order quantities, $\mathcal{R}_{a c}$ and $\delta \mathcal{D}_{c b}$, both symmetric at first order and containing a trace and a traceless part. The symmetric traceless part of this product is simply given by

$$
\chi\left(\mathcal{R}_{a c} \delta \mathcal{D}_{c b}\right)^{S T}=\chi\left(\mathcal{R}_{a b}\right)^{T} \frac{1}{2} \delta \mathcal{D}+\chi \frac{1}{2} \mathcal{R}\left(\delta \mathcal{D}_{a b}\right)^{T},
$$

where $\delta \mathcal{D}$ is the perturbed trace of the mapping $\mathcal{D}_{a b}$ and $\mathcal{R}$ is the trace of $\mathcal{R}_{a b}$. Note that the term $\chi \mathcal{R}_{a c}^{T} \delta \mathcal{D}_{c b}^{T}$ contains a trace and an antisymmetric part and thus contributes only to the second-order convergence and rotation. Using the expressions for ${ }_{2} \mathcal{R}$ and $\mathcal{R}$, eqs. (41) and (42), and those for ${ }_{2} \mathcal{D}$ and $\mathcal{D}$, eqs. (43) and (45), one obtains for the contribution of the second term to ${ }_{2} \mathcal{S}$,

$$
\begin{aligned}
& 2 \psi \not \partial^{2} \Psi-\frac{2}{\chi} \not \partial^{2} \Psi \int_{0}^{\chi} \mathrm{d} \chi^{\prime}\left(2 \Psi-\frac{\chi-\chi^{\prime}}{\chi^{\prime}} \bar{\partial} \not \partial \Psi\right) \\
& +2\left(2 \Psi_{, r}+\frac{1}{\chi} \bar{\partial} \not \partial \Psi+\chi \frac{\mathrm{d}^{2} \psi}{\mathrm{d} \chi^{2}}\right) \int_{0}^{\chi} \mathrm{d} \chi^{\prime} \frac{\chi-\chi^{\prime}}{\chi^{\prime}} \not \partial^{2} \Psi .
\end{aligned}
$$

The first line is $\chi_{2} \mathcal{R} \delta \mathcal{D}$ while the second is $\chi \mathcal{R}{ }_{2} \delta \mathcal{D}$.

The third term of $\mathcal{S}_{a b}$ is a pure trace so that it does not contribute to the shear. The traceless part of the fourth term of eq. (54) can be straightforwardly computed by noting that

$$
\frac{\mathrm{d} k^{0}}{\mathrm{~d} \chi}=-2 \frac{\mathrm{d} \phi}{\mathrm{d} \chi}+2 \dot{\Psi}
$$

Thus, its contribution to the source ${ }_{2} \mathcal{S}$ is

$$
4 \chi\left(-\frac{\mathrm{d} \phi}{\mathrm{d} \chi}+\dot{\Psi}\right) \int_{0}^{\chi} \mathrm{d} \chi^{\prime} \frac{1}{\chi^{\prime}} \not \partial^{2} \Psi .
$$

In order to express the last term of eq. (54) in terms of a spin-2 field we need to solve the geodesic equation at first order. We can solve $\mathrm{d} x^{i} / \mathrm{d} \chi=k^{i} / k^{0}$ making use of eq. (59). After integrating by parts this yields the geodesic deviation,

$$
\delta x^{i}=e_{r}^{i} \int_{0}^{\chi} d \chi^{\prime} 2 \Psi-e_{a}^{i} e_{a}^{j} \int_{0}^{\chi} d \chi^{\prime}\left(\chi-\chi^{\prime}\right) 2 \Psi_{, j} .
$$

The shift of $\mathcal{R}_{a b}, \delta\left(\mathcal{R}_{a b}\right)_{i}$, is simply given by the variation of $\Psi$ and $\mathrm{d}^{2} \psi / \mathrm{d} \chi^{2}$ along the geodesic in eq. (28). We only need to take the traceless part of $\delta\left(\mathcal{R}_{a b}\right)_{i}$. Thus we have

$$
\delta\left(\mathcal{R}_{a b}\right)_{i}^{T}=-e_{a}^{j} e_{b}^{k} 2 \Psi_{, i j k}
$$

which can be contracted with $\delta x^{i}$ of eq. (66). Note that, as we are varying directly the scalar Weyl potential $\Psi$, it was not necessary to introduce a covariant derivative on the sphere as in [46]. With the definitions and relations of Sec. 3 it is possible to verify that

$$
e_{+}^{i} e_{+}^{j} e_{+}^{k} X_{, i j k}=-\frac{1}{\chi^{3}} \not \partial^{3} X, \quad e_{+}^{i} e_{+}^{j} e_{-}^{k} X_{, i j k}=-\frac{1}{\chi^{3}} \not \partial \not \partial{ }^{2} X-\frac{4}{\chi^{2}}\left(\not \partial X_{, r}-\frac{1}{\chi} \not \partial X\right) .
$$


Using these relations and $e_{a}^{i} e_{a}^{j}=\left(e_{+}^{i} e_{-}^{j}+e_{-}^{i} e_{+}^{j}\right) / 2$ one finds, for this last contribution,

$$
\begin{aligned}
& 4\left(\frac{2}{\chi} \not \partial^{2} \Psi-\not \partial^{2} \Psi_{, r}\right) \int_{0}^{\chi} \mathrm{d} \chi^{\prime} \Psi \\
& +\frac{2}{\chi} \not \partial^{3} \Psi \int_{0}^{\chi} \mathrm{d} \chi^{\prime} \frac{\chi-\chi^{\prime}}{\chi^{\prime}} \bar{\partial} \Psi+\frac{2}{\chi} \not \partial \not^{2} \Psi \int_{0}^{\chi} \mathrm{d} \chi^{\prime} \frac{\chi-\chi^{\prime}}{\chi^{\prime}} \not \partial \Psi+8\left(\not \partial \Psi_{, r}-\frac{1}{\chi} \not \partial \Psi\right) \int_{0}^{\chi} \mathrm{d} \chi^{\prime} \frac{\chi-\chi^{\prime}}{\chi^{\prime}} \not \partial \Psi,
\end{aligned}
$$

where the first and second lines come from contracting with the first and second terms on the righthand side of eq. (66), respectively. Using the commutation rule for the spin raising and lowering operators [4],

$$
(\bar{\varnothing} \not \partial-\not \supset \bar{\phi})_{s} X=2 s_{s} X,
$$

it is convenient to rewrite the second term in the second line of eq. (69) as

$$
\frac{2}{\chi} \bar{\partial} \not \partial^{2} \Psi \int_{0}^{\chi} \mathrm{d} \chi^{\prime} \frac{\chi-\chi^{\prime}}{\chi^{\prime}} \not \partial \Psi=\frac{2}{\chi} \not \partial \bar{\partial} \not \partial \Psi \int_{0}^{\chi} \mathrm{d} \chi^{\prime} \frac{\chi-\chi^{\prime}}{\chi^{\prime}} \not \partial \Psi+\frac{4}{\chi} \not \partial \Psi \int_{0}^{\chi} \mathrm{d} \chi^{\prime} \frac{\chi-\chi^{\prime}}{\chi^{\prime}} \not \partial \Psi .
$$

Finally, combining eqs. (61), (63), (65), (69) and (71), replacing partial derivatives with respect to $r$ by using $\partial_{r}=\mathrm{d} / \mathrm{d} \chi-\partial_{\chi}$ (we remind the reader that $\dot{\Psi}=\partial_{\chi} \Psi$ ) and integrating the total derivatives by parts, we obtain

$$
\begin{aligned}
{ }_{2} \mathcal{D}\left(\chi_{S}\right)= & 2 \int_{0}^{\chi_{s}} d \chi \frac{\chi_{S}-\chi}{\chi} \not \partial\left[-\not \partial \Psi+\frac{1}{\chi}\left(\not \partial^{2} \Psi \int_{0}^{\chi} d \chi^{\prime} \frac{\chi-\chi^{\prime}}{\chi^{\prime}} \bar{\partial} \Psi+\bar{\partial} \not \partial \Psi \int_{0}^{\chi} d \chi^{\prime} \frac{\chi-\chi^{\prime}}{\chi^{\prime}} \not \partial \Psi\right)\right] \\
& +4 \int_{0}^{\chi_{s}} d \chi \frac{\chi_{S}-\chi}{\chi}\left[\frac{1}{2} \not \partial^{2} \Psi^{2}+\frac{1}{2} \psi\left(\chi_{S}\right) \not \partial^{2} \Psi-\Psi \frac{1}{\chi} \int_{0}^{\chi} d \chi^{\prime} \not \partial^{2} \Psi+\not \partial^{2}\left(\dot{\Psi} \int_{0}^{\chi} d \chi^{\prime} \Psi\right)\right. \\
& \left.+\not \partial \Psi \frac{1}{\chi} \int_{0}^{\chi} d \chi^{\prime} \frac{\chi-\chi^{\prime}}{\chi^{\prime}} \not \partial \Psi\right]-4 \int_{0}^{\chi_{S}} d \chi\left[\Psi \int_{0}^{\chi} d \chi^{\prime} \frac{1}{\chi^{\prime}} \not \partial^{2} \Psi+\frac{1}{\chi} \not \partial^{2}\left(\Psi \int_{0}^{\chi} d \chi^{\prime} \Psi\right)\right] \\
& +\frac{\chi_{S}}{2}{ }_{2} h\left(\chi_{S}\right)+\int_{0}^{\chi_{S}} d \chi\left[\frac{\chi_{S}-\chi}{\chi} \not \partial^{2}\left(\omega_{r}+\frac{1}{2} h_{r r}\right)+\frac{\chi_{S}}{\chi} \not \partial\left({ }_{1} \omega+{ }_{1} h_{r}\right)\right] .
\end{aligned}
$$

This is the solution of the Sachs equation. It is written in terms of spin raising and lowering operators $\not \partial$ and $\bar{\not}$, which are just the extensions on the sphere of the usual angular gradients: for small angles one can replace them by angular gradients $\vec{\nabla}_{e_{r}}$ transverse to the line of sight. The advantage of using this representation is that the eigenfunctions of these operators are simply spin-weighted spherical harmonics (see Appendix B).

In the small-angle approximation, i.e. when transverse scales are much smaller than radial scales, eq. (72) reduces to its first line. Indeed, in harmonics space the presence of the operators $\not \partial$ and $\bar{\phi}$ is associated to an $l$ factor. Thus, when observations are confined to large $l$ one gets a larger contribution from those terms containing more $\not \partial$ and $\bar{\phi}$ operators, such as the first line. The parentheses of the first line contain the usual couplings considered in the literature [21, 22, 23, 24, 25], i.e. the lens-lens correction and the correction to the Born approximation. Note that there are other 
couplings at play: the nonlinear growth of dark matter fluctuations induced by gravity on small scales introduces other second-order effects that are incorporated in the Weyl potential $\Psi 7$

In the small-angle approximation the last three lines of eq. (72) are suppressed by the ratio between the transverse scales probed and the longitudinal distances. However, for a full-sky survey where one probes larger scales they can become important. Let us list here these terms collecting them by their physical interpretation (for simplicity, integration over $\mathrm{d} \chi$ and $\mathrm{d} \chi^{\prime}$ will be omitted):

- An intrinsic contribution, integrated only once along the line of sight,

$$
2 \frac{\chi_{S}-\chi}{\chi} \not \partial^{2} \Psi^{2}(\chi)
$$

coming from the Riemann tensor at second order. This is a purely general relativistic effect of second-order gravity.

- A source-lens coupling,

$$
2 \frac{\chi_{S}-\chi}{\chi} \psi\left(\chi_{S}\right) \not \partial^{2} \Psi^{2}(\chi)
$$

which comes from the coupling between the lens and the curvature at the source, inducing a deformation of its shape. As we will see, this term is absent from the final expression of the reduced shear, as it cancels with an equivalent term coming from the corrections due to the denominator of eq. (17).

- Time delay-lens couplings,

$$
\begin{aligned}
& 4 \frac{\chi_{S}-\chi}{\chi}\left(-\Psi(\chi) \frac{1}{\chi} \not \partial^{2} \Psi\left(\chi^{\prime}\right)+\not \partial^{2} \dot{\Psi}(\chi) \Psi\left(\chi^{\prime}\right)+\dot{\Psi}(\chi) \not \partial^{2} \Psi\left(\chi^{\prime}\right)\right) \\
& -4\left(\Psi(\chi) \frac{1}{\chi^{\prime}} \not \partial^{2} \Psi\left(\chi^{\prime}\right)+\frac{1}{\chi} \not \partial^{2} \Psi(\chi) \Psi\left(\chi^{\prime}\right)+\frac{1}{\chi} \Psi(\chi) \not \partial^{2} \Psi\left(\chi^{\prime}\right)\right),
\end{aligned}
$$

which come from the coupling between the lens and the time delay that occurs during the longitudinal photon path.

- Deflection-deflection couplings,

$$
4 \frac{\chi_{S}-\chi}{\chi}\left(2 \not \partial \dot{\Psi}(\chi) \not \partial \Psi\left(\chi^{\prime}\right)+\not \partial \Psi(\chi) \frac{\chi-\chi^{\prime}}{\chi^{\prime}} \not \partial \Psi\left(\chi^{\prime}\right)\right)-\frac{8}{\chi} \not \partial \Psi(\chi) \not \partial \Psi\left(\chi^{\prime}\right),
$$

which are due to the couplings between two changes in the photon directions. Note that the photon deflection is described by a spin-1 field (the deflecting angle), i.e. a spin raising operator $\not \partial$ acting on a scalar. Taken alone it does not change the shear as it just affects all photons of the beam in the same way. However, the coupling of two deflections generate a spin-2 field which contributes to the shear. These corrections, as well as the time delay-lens ones, are integrated twice along the line of sight.

\footnotetext{
${ }^{7}$ To a large extent, these effects are those that are expected to dominate in the current surveys and that lead to detectable effects $[21,47,48$.
} 
The last line of eq. (72) contains the effects induced by vector and tensor modes generated at second order. As vectors and tensors are already second-order quantities, they enter linearly in this expression. The vector component enters only through terms integrated along the line of sight. As the tensor component is a spin-2 field it induces also a boundary term, which accounts for the distortion of the shape at the source. The integrated contributions from the tensor component agree with what was found in [49, 50]. Note that, at second order, the separation into scalars, vectors and tensors done here is gauge dependent. Indeed, we expect all these different contributions to give comparable effects to second-order observables such as the bispectrum. This is similar, for instance, to what happens when one computes the CMB bispectrum on large angular scales [14]. Finally, note that our final result (72) cannot be written as the action of $\not \not^{2}$ on a scalar quantity. Thus, the shear will contain also $B$ modes (see Appendix (B) ).

\subsection{Testing the solution}

As the reader has certainly realized, the derivation of eq. (72) is extremely tedious and involves many steps. Thus, it is important to develop tests in order to check this equation and, in particular, the new nonlinear couplings (73)-(76). One implicit check is that ${ }_{2} \mathcal{D}$ behaves as a spin-2 field under rotation of the screen basis. This is automatically ensured by the use of the operators $\not \partial$ and $\bar{\phi}$ instead of the angular derivatives. Furthermore, we can check the terms in eqs. (73) - (75) by studying specific cases where part of our calculation can be pursued nonperturbatively. For instance, we can study the shear at linear order in a universe with constant curvature. In the limit where the curvature is small we must recover the couplings between the curvature and the gravitational potential given in eqs. (73)-(75) . As we will see, such a strategy can be generalized to other cases. Unfortunately we were not able to develop an analogous test for the terms appearing in eq. (76).

\subsubsection{Couplings from perturbing the spatial curvature}

Let us consider a perturbed nonexpanding FLRW spacetime with constant curvature $K$ and metric

$$
d s^{2}=-(1+2 \phi) \mathrm{d} \eta^{2}+\frac{1}{\left(1+\psi_{K}\right)^{2}}\left(d r^{2}+r^{2} d \Omega^{2}\right),
$$

with

$$
\phi=\phi\left(x^{\mu}\right), \quad \psi_{K}=\frac{K r^{2}}{4},
$$

where we have perturbed only the 00 part of the metric. At linear order, the spin-2 mapping field ${ }_{2} \mathcal{D}$ for this metric is (see for instance [21])

$$
{ }_{2} \mathcal{D}_{K}\left(\chi_{S}\right)=-\int_{0}^{\chi_{S}} \mathrm{~d} \chi \frac{D_{A}\left(\chi_{S}-\chi\right)}{D_{A}(\chi)} \not \partial^{2} \phi
$$


where $D_{A}(\chi)$ is the angular diameter distance given by eq. (47). Equation (79) is just the generalization of eq. (43), where $D_{A}(\chi)=\chi$, to a constant curved FLRW universe. Since $\psi_{K}$ does not depend on the angles, $\not \partial^{2} \psi_{K}=0$ and thus only $\not \partial^{2} \phi$ appears on the right-hand side of this equation.

For a small curvature $K$ we can expand eq. (79) at first order in $\psi_{K}$. The angular diameter distance (47) reads $D_{A}(\chi) \simeq \chi-K \chi^{3} / 6$. Furthermore, we have to evaluate $\phi$ on the geodesic solution for a curved universe, i.e. at $r(\chi)=\chi+K \chi^{3} / 12$, which yields

$$
\not \partial^{2} \phi(r(\chi), \theta, \phi)=\not \partial^{2} \phi(\chi, \theta, \phi)+\frac{K \chi^{3}}{12} \not \partial^{2} \phi, r(\chi, \theta, \phi) \text {. }
$$

Plugging these expressions into eq. (79), replacing the derivative with respect to $r$ using $\partial_{r}=$ $\mathrm{d} / \mathrm{d} \chi-\partial_{\chi}$ and integrating by parts we obtain, up to first order in $\psi_{K}$,

$$
{ }_{2} \mathcal{D}_{K}=-\int_{0}^{\chi_{S}} \mathrm{~d} \chi\left[\frac{\chi_{S}-\chi}{\chi} \not \partial^{2} \phi-\frac{K}{12}\left(\frac{2 \chi_{S}^{3}}{\chi}-3 \chi^{2}-6 \chi_{S}^{2}+6 \chi \chi_{S}\right) \not \partial^{2} \phi-\frac{K}{12}\left(\chi_{S}-\chi\right) \chi^{2} \not \partial^{2} \dot{\phi}\right] .
$$

The last two terms on the right-hand side of this equation can be seen as "second-order" corrections to the first-order expression (43), of order $\sim \mathcal{O}\left(\phi \psi_{K}\right)$, due to the coupling between the gravitational potential $\phi$ and the curvature perturbation $\psi_{K}$. These corrections are already incorporated in our second-order expression (72). Indeed, by replacing $\Psi=\left(\phi+\psi_{K}\right) / 2$ in this equation, neglecting second-order terms of order $\sim \mathcal{O}\left(\phi^{2}\right)$ but keeping those of order $\sim \mathcal{O}\left(\phi \psi_{K}\right)$ one finds, after integrating by parts, eq. (81).

This calculation can be extended to a spacetime with radial-dependent curvature, i.e. $\psi_{K}(r)$ a generic function of $r$. In this case the spin-2 mapping ${ }_{2} \mathcal{D}$ is given by

$$
{ }_{2} \mathcal{D}_{K}\left(\chi_{S}\right)=-\int_{0}^{\chi_{S}} \mathrm{~d} \chi \frac{G\left(\chi_{S}, \chi\right)}{D_{A}(\chi)} \not \not^{2} \phi,
$$

where the Green's function $G\left(\chi_{S}, \chi\right)$ and the angular diameter distance $D_{A}(\chi)$ can be derived from using eq. (19) in a homogeneous universe with spatial curvature $\psi_{K}$. In this case the trace of eq. (19) becomes

$$
\frac{\mathrm{d}^{2} D_{A}(\chi)}{\mathrm{d} \chi^{2}}=R(r) D_{A}(\chi)
$$

where, at first order in $\psi_{K}, R=-\psi_{K, r} / r-\psi_{K, r r}$, implying

$$
\psi_{K}=-\int^{\chi} \mathrm{d} \chi^{\prime} / \chi^{\prime} \int^{\chi^{\prime}} \mathrm{d} \chi^{\prime \prime} \chi^{\prime \prime} R\left(\chi^{\prime \prime}\right) .
$$

The two solutions of eq. (83), $D_{A}(\chi)$ and $C(\chi)$, are determined through their initial conditions, i.e. $D_{A}(\chi) \rightarrow \chi$ and $C(\chi) \rightarrow 1+\mathcal{O}\left(\chi^{2}\right)$, so that

$$
\begin{aligned}
D_{A}(\chi) & =\chi+\int_{0}^{\chi} \mathrm{d} \chi^{\prime} \int_{0}^{\chi^{\prime}} \mathrm{d} \chi^{\prime \prime} \chi^{\prime \prime} R\left(\chi^{\prime \prime}\right), \\
C(\chi) & =1+\int_{0}^{\chi} \mathrm{d} \chi^{\prime} \int_{0}^{\chi^{\prime}} \mathrm{d} \chi^{\prime \prime} R\left(\chi^{\prime \prime}\right) .
\end{aligned}
$$


The Green's function is then given by

$$
G\left(\chi_{S}, \chi\right)=D_{A}\left(\chi_{S}\right) C(\chi)-D_{A}(\chi) C\left(\chi_{S}\right)
$$

Evaluating $\not \partial^{2} \phi$ on the geodesic solution for a curved universe, and replacing eqs. (85) and (87) into (82) we obtain, at first order in $\phi$ and $\psi_{K}$ but keeping terms of order $\sim \mathcal{O}\left(\phi \psi_{K}\right)$,

$$
\begin{aligned}
{ }_{2} \mathcal{D}_{K}\left(\chi_{S}\right)= & -\int_{0}^{\chi_{S}} \mathrm{~d} \chi \not^{2} \phi\left[\frac{\chi_{S}-\chi}{\chi}\left(1+\int_{0}^{\chi} \mathrm{d} \chi^{\prime} \int_{0}^{\chi^{\prime}} \mathrm{d} \chi^{\prime \prime} R\left(\chi^{\prime \prime}\right)-\frac{1}{\chi} \int_{0}^{\chi} \mathrm{d} \chi^{\prime} \int_{0}^{\chi^{\prime}} \mathrm{d} \chi^{\prime \prime} \chi^{\prime \prime} R\left(\chi^{\prime \prime}\right)\right)\right. \\
& \left.-\int_{\chi}^{\chi_{S}} \mathrm{~d} \chi^{\prime} \int_{0}^{\chi^{\prime}} \mathrm{d} \chi^{\prime \prime} R\left(\chi^{\prime \prime}\right)+\frac{1}{\chi} \int_{\chi}^{\chi_{S}} \mathrm{~d} \chi^{\prime} \int_{0}^{\chi^{\prime}} \mathrm{d} \chi^{\prime \prime} \chi^{\prime \prime} R\left(\chi^{\prime \prime}\right)\right] \\
& -\int_{0}^{\chi_{S}} \mathrm{~d} \chi \frac{\chi_{S}-\chi}{\chi} \not \partial^{2} \phi_{, r} \int_{0}^{\chi} \psi_{K} \mathrm{~d} \chi^{\prime} .
\end{aligned}
$$

We have checked that, after integration by part of the last line, our general expression (72) reproduces this peculiar case.

\subsubsection{Reparametrization invariance under a time shift}

Until now we have tested the couplings between the 00 metric perturbation $\phi\left(x^{\mu}\right)$ and a radialdependent spatial curvature $\psi_{K}(r)$. Analogously, we can test the nonlinear couplings between the spatial metric perturbation $\psi\left(x^{\mu}\right)$ and a 00 metric perturbation which depends only on time, $\phi_{T}(\eta)$. Such a perturbation can be reabsorbed into the time coordinate through a homogeneous shift of the time $\mathrm{d} \chi=\mathrm{d} \tilde{\chi}\left(1+\phi_{T}(\tilde{\chi})\right)$. As a homogeneous time shift does not change the gauge, we expect our expression (72) to be invariant under a first-order coordinate change

$$
\chi=\tilde{\chi}+\int_{0}^{\tilde{\chi}} \mathrm{d} \tilde{\chi}^{\prime} \phi_{T}, \quad x^{i}=\tilde{x}^{i}
$$

Let us check that this is the case. At first order in $\phi_{T}$, under this coordinate transformation the metric perturbations change as $\phi(\chi)=\tilde{\phi}(\tilde{\chi})-\phi_{T}(\tilde{\chi})$ and $\psi(\chi)=\tilde{\psi}(\tilde{\chi})$. Evaluating $\Psi$ on the geodesic $r=\chi=\tilde{\chi}+\int_{0}^{\tilde{\chi}} \mathrm{d} \tilde{\chi}^{\prime} \phi_{T}$, the first term of eq. (72) transforms as

$$
\begin{aligned}
-2 \int_{0}^{\chi_{S}} \mathrm{~d} \chi \frac{\chi_{S}-\chi}{\chi} \not \partial^{2} \Psi= & -2 \int_{0}^{\tilde{\chi}_{S}} \mathrm{~d} \tilde{\chi} \frac{\tilde{\chi}}{\tilde{\chi}_{S}-\tilde{\chi}} \\
& -2 \int_{0}^{\tilde{\chi}} \mathrm{d} \tilde{\chi} \tilde{\chi} \frac{1}{\tilde{\chi}} \not \partial^{2} \tilde{\Psi} \int_{0}^{\tilde{\chi}} d \tilde{\chi}^{\prime} \phi_{T}+2 \int_{0}^{\tilde{\chi}} \mathrm{d} \tilde{\chi} \frac{\tilde{\chi} \tilde{\chi}_{S}-\tilde{\chi}}{\tilde{\chi}} \not \not^{2} \dot{\tilde{\Psi}} \int_{0}^{\tilde{\chi}} d \tilde{\chi}^{\prime} \phi_{T} .
\end{aligned}
$$

One can check that replacing $\Psi(\chi)=\tilde{\Psi}(\tilde{\chi})-\phi_{T}(\tilde{\chi})$ and $\chi=\tilde{\chi}$ into the second-order terms of eq. (72) exactly cancels the second line of eq. (90), leaving ${ }_{2} \mathcal{D}$ invariant under the transformation (89). 


\subsubsection{Reparametrization under conformal transformation}

In Appendix $\mathrm{A}$ we show that under the conformal transformation of the metric $g_{\mu \nu} \rightarrow \Omega^{2}\left(x^{\mu}\right) g_{\mu \nu}$ the mapping $\mathcal{D}_{a b}$ transforms as

$$
\mathcal{D}_{a b} \rightarrow \Omega \mathcal{D}_{a b}
$$

If the conformal factor is just $\Omega=1+\delta \Omega$, where $\delta \Omega$ is a small perturbation of order $\Psi$, this conformal transformation is equivalent, at first order in $\delta \Omega$, to a redefinition of the potentials in the metric,

$$
\psi \rightarrow \psi-\delta \Omega, \quad \phi \rightarrow \phi+\delta \Omega
$$

Since, as expected, the Weyl potential $\Psi=(\phi+\psi) / 2$ does not change under this transformation, in eq. (72) only the boundary term proportional to $\psi\left(\chi_{S}\right)$ is not invariant. Transforming this term according to (92) yields a contribution to ${ }_{2} \mathcal{D}\left(\chi_{S}\right)$ in the new metric,

$$
{ }_{2} \mathcal{D}\left(\chi_{S}\right) \rightarrow{ }_{2} \mathcal{D}\left(\chi_{S}\right)-2 \delta \Omega\left(x_{S}^{\mu}\right) \int_{0}^{\chi_{S}} d \chi \frac{\chi_{S}-\chi}{\chi} \not \not^{2} \Psi
$$

This is exactly what we expect from the conformal transformation ${ }_{2} \mathcal{D} \rightarrow(1+\delta \Omega)_{2} \mathcal{D}$.

\subsection{The reduced shear}

As mentioned in Sec. 2, the quantity that we measure is the reduced shear, which is given by the ratio between the spin-2 anisotropic mapping ${ }_{2} \mathcal{D}$ and the trace $\mathcal{D}$, eq. (17). Expanding this equation up to second order using that $\mathcal{D}=2 \chi_{S}+\delta \mathcal{D}$ we obtain for the reduced shear

$$
g=-\frac{{ }_{2} \mathcal{D}}{2 \chi_{S}}+\frac{{ }_{2} \mathcal{D} \delta \mathcal{D}}{\left(2 \chi_{S}\right)^{2}}
$$

where ${ }_{2} \mathcal{D}$ in the first term on the right-hand side is given by eq. (72) and, from eqs. (43) and (45), the second-order correction on the right-hand side is given by

$$
\begin{aligned}
\frac{{ }_{2} \mathcal{D} \delta \mathcal{D}}{\left(2 \chi_{S}\right)^{2}}= & \int_{0}^{\chi_{S}} d \chi \frac{\chi_{S}-\chi}{\chi_{\chi_{S}}} \bar{\phi} \not \partial \Psi \int_{0}^{\chi_{S}} d \chi^{\prime} \frac{\chi_{S}-\chi^{\prime}}{\chi^{\prime} \chi_{S}} \not \partial^{2} \Psi \\
& +\psi\left(\chi_{S}\right) \int_{0}^{\chi_{S}} d \chi \frac{\chi_{S}-\chi}{\chi \chi_{S}} \not \not^{2} \Psi-2 \int_{0}^{\chi_{S}} d \chi \Psi \int_{0}^{\chi_{S}} d \chi^{\prime} \frac{\chi_{S}-\chi^{\prime}}{\chi^{\prime} \chi_{S}^{2}} \not \partial^{2} \Psi
\end{aligned}
$$

The first line is the usual correction to the reduced shear due to the coupling between the convergence and the shear [28, 29]. The two corrections in the second line are negligible in the small-angle approximation. The term proportional to $\psi\left(\chi_{S}\right)$ comes from the coupling between the lens and the curvature perturbation at the source contained in the trace of $\mathcal{D}_{a b}$, see eq. (45). Note that it cancels with the one in the expression of ${ }_{2} \mathcal{D}$. Indeed, as both the isotropic and anisotropic part of $\mathcal{D}_{a b}$ change according to eq. (91) under conformal transformation, we expect the reduced shear, which is their ratio, to depend only on the Weyl potential $\Psi$, which is invariant under conformal transformation. The last term in the second line is the coupling between the time delay contained in $\mathcal{D}$ with the lens. These terms are thus of the same order as those discussed in eqs. (73) $-(75)$ 
The expression of the reduced shear above is given taking the source at constant conformal time $\eta_{S}$. However, in order to relate this quantity to observations we need to compute it using a constant redshift $z_{S}$ for the source, given by

$$
z_{S}=\frac{k_{S}^{\alpha} v_{S \alpha}}{k_{O}^{\alpha} v_{O \alpha}}-1
$$

As in the Poisson gauge the $z=$ const hypersurfaces do not coincide with the $\eta=$ const hypersurfaces, the redshift $z_{S}$ is not homogeneous and we expect a correction to eq. (94) coming from the coupling between the perturbed redshift plane of the source and the lens. As explained in Sec. 2, eq. (20), the reduced shear at constant redshift is given by

$$
g_{z}=g+\delta g_{z}, \quad \delta g_{z} \equiv-\frac{\mathrm{d} g}{\mathrm{~d} \chi_{S}} \frac{\mathrm{d} \chi_{S}}{\mathrm{~d} z_{S}} \delta z_{S},
$$

where $\delta z_{S}$ is the perturbation of the redshift (96). We must now reintroduce the expansion of the Universe. By taking the derivative with respect to $\chi_{S}$ of the linear expression for $g$, eq. (44), we find

$$
\frac{\mathrm{d} g}{\mathrm{~d} \chi_{S}} \frac{\mathrm{d} \chi_{S}}{\mathrm{~d} z_{S}}=\frac{1}{\chi_{S}^{2} H_{S}} \int_{0}^{\chi_{S}} d \chi \not^{2} \Psi
$$

where $H$ is the Hubble rate defined from the cosmic time $t, \mathrm{~d} t=-a \mathrm{~d} \chi$, as $H \equiv \frac{1}{a} \frac{\mathrm{d} a}{\mathrm{~d} t}$.

To compute $\delta z_{S}$ we can perturb at first order eq. (96) using the expression for $k^{0}$ given in eq. (27). Setting to zero the perturbations of the metric and of the velocity at the observer position, we obtain

$$
\delta z_{S}=-\left(1+z_{S}\right)\left(\phi\left(\chi_{S}\right)+\left.\mathbf{e}_{r}\right|_{\chi_{S}} \cdot \mathbf{v}_{S}-2 \int_{0}^{\chi_{S}} \mathrm{~d} \chi \dot{\Psi}\right) .
$$

In the three terms on the right-hand side of this equation one recognizes the Sachs-Wolfe, the Doppler and the integrated Sachs-Wolfe effects contributing to the photon redshift perturbation. Finally, combining the expression (98) and the redshift perturbation (99) we obtain for the redshift correction (97),

$$
\delta g_{z}=\frac{1+z_{S}}{\chi_{S}^{2} H_{S}}\left(\phi\left(\chi_{S}\right)+\left.\mathbf{e}_{r}\right|_{\chi_{S}} \cdot \mathbf{v}_{S}-2 \int_{0}^{\chi_{S}} \mathrm{~d} \chi \dot{\Psi}\right) \int_{0}^{\chi_{S}} d \chi \not^{2} \Psi
$$

The observed reduced shear at constant redshift becomes then 8

$$
g_{z}=-\frac{{ }_{2} \mathcal{D}}{2 \chi_{S}}+\frac{{ }_{2} \mathcal{D} \delta \mathcal{D}}{\left(2 \chi_{S}\right)^{2}}+\delta g_{z}
$$

where the first, the second and the third terms on the right-hand side are respectively given by eqs. (72), (95) and (100). This is the main result of this article.

\footnotetext{
${ }^{8}$ Note that the only effect of the transverse velocity of the source is to modify the direction under which the galaxy is observed. Thus, it has no effect on the shear.
} 


\section{Conclusion}

In this article we have derived the expression of the reduced cosmic shear up to second order in the perturbations with full-sky validity. Our main result is summarized in eq. (101). As it is expressed in terms of spin- 2 operators on the sphere it can be decomposed as sum of spin-weighted spherical harmonics on the sky. Indeed, this description ensures that our observable has a genuine spin-2 behavior on the celestial sphere.

Our result is written in terms of the metric perturbations in the generalized Poisson gauge. These are the scalar potentials $\phi$ and $\psi$ and the vector and tensor components of the metric generated at second order, respectively $\omega_{i}$ and $h_{i j}$. Let us first comment on the first two terms on the right-hand side of eq. (101). Remarkably, the contribution from scalar perturbations from the sum of these two terms can be expressed in terms of the Weyl potential $\Psi=(\phi+\psi) / 2$ only. As explained, this is due to the fact that null geodesics are conformally invariant. These two terms contain the well-known second-order corrections due to lens-lens coupling and departure from the Born approximation, which dominate in the small-angle approximation. On larger angular scales new couplings become important. These are an intrinsic contribution which is a purely general relativistic effect at second order, a coupling between the gravitational potential at the source with the lens and corrections due to couplings between the lens and the photon time-delay. We have checked that these contributions can be independently reconstructed from the calculation of the shear at first order in a universe with a radially dependent spatial curvature. Other checks, such as the invariance under a homogeneous time shift and a conformal transformation can be used to verify the validity of these new corrections. Another scalar correction appears in the form of products of two spin-1 fields and comes from the couplings between two photon deflections. Finally, besides the scalar contributions, the shear gets a contribution from spin-2 quantities defined from the vector and tensor components of the metric generated at second-order. Note that the separation between all these contributions is not gauge invariant.

In Poisson gauge, the correction due to the coupling between the photon redshift perturbation and the lens cannot be written in terms of $\Psi$ only. Indeed, the integrated contribution to the photon redshift - the integrated Sachs-Wolfe effect - is a time integral over $\dot{\Psi}$ but the intrinsic contributions - Sachs-Wolfe and Doppler effects - are expressed in terms of the Newtonian gravitational potential and the velocity along the line of sight and do not depend on $\Psi$ only.

We are now in the position to explore the phenomenological consequences of these results in view of the future (partially) full-sky lensing surveys. In particular, the new corrections that we have computed should become relevant in deriving the lensing bispectrum on large angular scales. For instance, to compute the bispectrum in the squeezed limit one needs to take one of the three modes to be much smaller than the other two, corresponding to angular scales comparable to the depth of the survey. As the lensing is a cumulative effect integrated along the line of sight, it is difficult, 
at this stage, to precisely guess the relative importance of the various contributions. In particular, although the lens-lens coupling terms are a priori larger by a factor $\sim l^{2}$ compared to the others, they may be damped by geometrical factors. We leave these investigations for the future.

\section{Ackowledgements}

It is a pleasure to thank Chiara Caprini for useful discussions and collaboration in the early stage of the preparation of this article and Nicolas Van de Rijt for spotting few typos. C.B. and F.V. acknowledge support from the EU Marie Curie Research and Training network UniverseNet (MRTNCT-2006-035863).

\section{A Conformal relations}

We consider two conformally related metrics $\tilde{g}_{\alpha \beta}=\Omega^{2}\left(x^{\mu}\right) g_{\alpha \beta}$. We want to show that the corresponding Jacobi mappings computed using eq. (10) with the respective metrics are related by $\tilde{\mathcal{D}}_{a b}=\Omega \mathcal{D}_{a b}$. We can decompose the Riemann tensor in terms of the Ricci tensor $R_{\alpha \beta}$ and the Weyl tensor $C_{\alpha \beta \rho \sigma}$ as 34

$$
R_{\alpha \beta \gamma \delta}=C_{\alpha \beta \gamma \delta}+g_{\alpha[\gamma} R_{\delta] \beta}-g_{\beta[\gamma} R_{\delta] \alpha}-\frac{1}{6} R g_{\alpha[\gamma} g_{\delta] \beta}
$$

where the brackets in the indices denote antisymmetrization. With this decomposition, the definition of $\mathcal{R}_{a b}$, eq. (7), simply yields

$$
\mathcal{R}_{a b}=C_{\alpha \beta \gamma \delta} n_{a}{ }^{\alpha} k^{\beta} k^{\gamma} n_{b}{ }^{\delta}-\frac{1}{2} \delta_{a b} R_{\beta \gamma} k^{\beta} k^{\gamma},
$$

where we have used the normalization properties of $n_{a}^{\mu}$ and $k^{\mu}$.

Using this expression, let us study how $\mathcal{R}_{a b}$ transforms under a conformal transformation. As $n_{a}^{\mu}$ is normalized to unity it transforms as $\tilde{n}_{a}^{\mu}=\Omega^{-1} n_{a}^{\mu}$. If $\lambda$ is an affine parameter of the null geodesic in the metric $g_{\alpha \beta}$, then the affine parameter computed using the metric $\tilde{g}_{\alpha \beta}$ is related to $\lambda$ by $\mathrm{d} \tilde{\lambda}=\Omega^{2} \mathrm{~d} \lambda$ [34]. Thus, $\tilde{k}^{\mu}=\Omega^{-2} k^{\mu}$. Furthermore, as the Weyl tensor with one upper index $C_{\alpha \beta \delta} \gamma$ is invariant under conformal transformation, the first term of (103) transforms as

$$
\tilde{C}_{\alpha \beta \gamma \delta} \tilde{n}_{a}^{\alpha} \tilde{k}^{\beta} \tilde{k}^{\gamma} \tilde{n}_{b}^{\delta}=\Omega^{-4} C_{\alpha \beta \gamma \delta} n_{a}^{\alpha} k^{\beta} k^{\gamma} n_{b}^{\delta}
$$

The Ricci tensor transforms as 34

$$
\tilde{R}_{\alpha \gamma}=R_{\alpha \gamma}-2 \nabla_{\alpha} \nabla_{\gamma} \ln \Omega-g_{\alpha \gamma} g^{\delta \sigma} \nabla_{\delta} \nabla_{\sigma} \ln \Omega+2\left(\nabla_{\alpha} \ln \Omega\right) \nabla_{\gamma} \ln \Omega-2 g_{\alpha \gamma} g^{\delta \sigma}\left(\nabla_{\delta} \ln \Omega\right) \nabla_{\sigma} \ln \Omega
$$


Projecting this expression by $\tilde{k}^{\alpha} \tilde{k}^{\gamma}$, the two terms proportional to $g_{\alpha \gamma}$ vanish because of the null condition of the photon wave vector, while the covariant derivatives can be written as derivatives along the null geodesic. Using the geodesic equation one obtains

$$
\tilde{R}_{\beta \gamma} \tilde{k}^{\beta} \tilde{k}^{\gamma}=\Omega^{-4}\left[R_{\beta \gamma} k^{\beta} k^{\gamma}-2 \frac{\mathrm{d}^{2} \ln \Omega}{\mathrm{d} \lambda^{2}}+2\left(\frac{\mathrm{d} \ln \Omega}{\mathrm{d} \lambda}\right)^{2}\right] .
$$

Thus, from eq. (103) $\mathcal{R}_{a b}$ transforms as

$$
\tilde{\mathcal{R}}_{a b}=\Omega^{-4}\left[\mathcal{R}_{a b}+\frac{\mathrm{d}^{2} \ln \Omega}{\mathrm{d} \lambda^{2}} \delta_{a b}-\left(\frac{\mathrm{d} \ln \Omega}{\mathrm{d} \lambda}\right)^{2} \delta_{a b}\right] .
$$

Finally, using this transformation it is easy to show that if $\mathcal{D}_{a b}$ is the solution of

$$
\frac{\mathrm{d}^{2}}{\mathrm{~d} \lambda^{2}} \mathcal{D}_{a b}=\mathcal{R}_{a c} \mathcal{D}_{c b}
$$

then $\tilde{\mathcal{D}}_{a b}=\Omega \mathcal{D}_{a b}$ is the solution of the corresponding equation for the metric $\tilde{g}_{\mu \nu}$,

$$
\frac{\mathrm{d}^{2}}{\mathrm{~d} \tilde{\lambda}^{2}} \tilde{\mathcal{D}}_{a b}=\tilde{\mathcal{R}}_{a c} \tilde{\mathcal{D}}_{c b}
$$

The relation between $\mathcal{D}_{a b}$ and $\tilde{\mathcal{D}}_{a b}$ can be easily understood by noting that $\mathcal{D}_{a b}$ relates distances at the source, that scale like $\Omega$, to angles at the observer that are invariant under a conformal transformation.

\section{B Spin operators on the sphere}

The construction of spin fields can be easily done on a plane identified with the complex plane of coordinates $z=x+\mathrm{i} y$. Let us consider a complex field ${ }_{s} X(z)$ whose value depends on $z$. This field will be said to have spin- $s$ if its value is changed in $e^{\mathrm{i} s \alpha}{ }_{s} X(z)$ after a rotation of angle $\alpha$. For instance, if ${ }_{0} X(z)$ is a scalar (i.e. a spin-0) field, then

$$
{ }_{1} X(z)=\partial_{x_{0}} X(z)+\mathrm{i} \partial_{y_{0}} X(z)
$$

is a spin-1 field. This relation can alternatively be written as ${ }_{1} X(z)=2 \partial_{z}{ }_{0} X(z)$, where the partial derivative is to be taken for a fixed value of $\bar{z}$. In general, the successive application of the operator $\not \partial \equiv 2 \partial_{z}$ leads to the construction of spin-s fields. Equivalently, the operator $\bar{\phi} \equiv 2 \partial_{\bar{z}}$ lowers the spin by one. In the context of standard first-order lensing theory, the complex shear field $\gamma=\gamma_{1}+\mathrm{i} \gamma_{2}$ is a spin-2 field that derives from the projected potential $\psi$, i.e. $\gamma=\not \partial^{2} \psi$.

This construction can be extended to the sphere when one does not want to rely on the smallangle approximation. The early elements of such a construction date back to [42, 40]. In general, one is naturally led to introduce the Euler angles $(\theta, \varphi, \alpha)$ so that the coordinates of a point on the unit sphere are $(\sin \theta \cos \varphi, \sin \theta \sin \varphi, \cos \theta)$. To each of these points one can associate a radial vector $\mathbf{e}_{r}$ 
and two tangential vectors $\mathbf{e}_{1}$ and $\mathbf{e}_{2}$ that can be conveniently chosen along the lines $\varphi=$ const and $\theta=$ const respectively if $\alpha=0$. The angle $\alpha$ then corresponds to a rotation around the axis $\mathbf{e}_{r}$ that rotates $\mathbf{e}_{\theta}$ and $\mathbf{e}_{\varphi}$ with an angle $\alpha$.

As for the plane, a spin- $s$ field is such that its phase varies as $s \alpha$, by rotation of an angle $\alpha$. The construction of the operators $\not \partial$ and $\bar{\phi}$ relies on the use of the complex stereographic coordinates,

$$
\zeta=\cot \left(\frac{\theta}{2}\right) \exp (\mathrm{i} \varphi)
$$

which map the sphere onto the complex plane. More specifically it can be shown that the expressions of the operators $\not \partial$ and $\bar{\phi}$ depend explicitly on the spin of the field ${ }_{s} X$ to which they are applied,

$$
\not_{s} X=2 P^{1-s} \partial_{\zeta} P_{s}^{s} X \quad \text { and } \quad \bar{\partial}_{s} X=2 P^{1+s} \partial_{\bar{\zeta}} P^{-s}{ }_{s} X
$$

where $P=\frac{1}{2}(1+\zeta \bar{\zeta})$. Expressing these operators in terms of $\theta$ and $\varphi$, one finds

$$
\not_{s} X=-(\sin \theta)^{s}\left(\partial_{\theta}+\mathrm{i} \csc \theta \partial_{\varphi}\right)(\sin \theta)^{-s}{ }_{s} X \text { and } \bar{\partial}_{s} X=-(\sin \theta)^{-s}\left(\partial_{\theta}-\mathrm{i} \csc \theta \partial_{\varphi}\right)(\sin \theta)^{s}{ }_{s} X \text {. }
$$

In analogy to the case of the plane, the Laplacian operator formally reads $\Delta=\bar{\phi} \not \partial$. Note however that the relation $\bar{\phi} \not \partial=\not \supset \bar{\phi}$ holds only when the operators act on a scalar field. In general we have, $(\bar{\not} \not \partial-\not \supset \bar{\not})_{s} X=2 s_{s} X$.

Spherical harmonics, $Y_{l}^{m}(\theta, \varphi)$, are spin-0 functions that are the eigenfunctions of the Laplacian with eigenvalue $-l(l+1)$, i.e. $\Delta Y_{l}^{m}(\theta, \varphi)=-l(l+1) Y_{l}^{m}(\theta, \varphi)$ with $\partial_{\varphi} Y_{l}^{m}(\theta, \varphi)=i m Y_{l}^{m}(\theta, \varphi)$ and with a specific normalization. The orthogonality relation,

$$
\int \mathrm{d}^{2} \Omega Y_{l^{\prime}}^{m^{\prime}}(\theta, \varphi) Y_{l}^{m *}(\theta, \varphi)=\delta_{l^{\prime} l} \delta_{m^{\prime} m}
$$

is the key property that makes it possible to decompose any function into spherical harmonics.

In general spin- $s$ fields are decomposed on the basis of the spin-weighted spherical harmonics, that can be obtained through the application of the operator $\not \partial$ and $\bar{\not}$ on the spherical harmonics. More specifically, we define ${ }_{s} Y_{l}^{m}(\theta, \varphi)$ with

$$
\begin{aligned}
{ }_{s} Y_{l}^{m}(\theta, \varphi) & =\left[\frac{(l-s) !}{(l+s) !}\right]^{1 / 2} \not \partial^{s} Y_{l}^{m}(\theta, \varphi), \quad(0 \leq s \leq l), \\
{ }_{s} Y_{l}^{m}(\theta, \varphi) & =\left[\frac{(l+s) !}{(l-s) !}\right]^{1 / 2}(-1)^{s} \bar{\phi}^{-s} Y_{l}^{m}(\theta, \varphi), \quad(-l \leq s \leq 0) .
\end{aligned}
$$

The spin-weighted spherical harmonics obey the following relations,

$$
\begin{aligned}
{ }_{s} Y_{l}^{m *}(\theta, \varphi) & =(-1)^{m}{ }_{-s} Y_{l}^{-m}(\theta, \varphi), \\
\bar{\partial} \not{ }_{s} Y_{l}^{m}(\theta, \varphi) & =-(l-s)(l+s+1){ }_{s} Y_{l}^{m}(\theta, \varphi), \\
\int \mathrm{d}^{2} \Omega{ }_{s} Y_{l^{\prime}}^{m^{\prime}}(\theta, \varphi){ }_{s} Y_{l}^{m *}(\theta, \varphi) & =\delta_{l^{\prime} l} \delta_{m^{\prime} m}, \\
\sum_{l m}{ }_{s} Y_{l}^{m}\left(\theta^{\prime}, \varphi^{\prime}\right)_{s} Y_{l}^{m *}(\theta, \varphi) & =\delta\left(\varphi-\varphi^{\prime}\right) \delta\left(\cos \theta-\cos \theta^{\prime}\right),
\end{aligned}
$$


with $\int \mathrm{d}^{2} \Omega \equiv \int_{0}^{2 \pi} d \varphi \int_{-1}^{1} d \cos \theta$.

One usually defines two scalars, $E$ and $B$, associated to the spin-2 shear $\gamma$. To do that, one decomposes the shear $\gamma$ and its complex conjugate $\gamma^{*}$ as,

$$
\gamma(\theta, \varphi)=\sum_{l m}{ }_{2} a_{l m} Y_{l}^{m}(\theta, \varphi) \quad \text { and } \quad \gamma^{*}(\theta, \varphi)=\sum_{l m}{ }_{2} a_{l m-2} Y_{l}^{m}(\theta, \varphi)
$$

As in the context of CMB polarization (see [51]), $E$ and $B$ can be defined through their harmonic decomposition,

$$
E(\theta, \varphi)=-\frac{1}{2} \sum_{l m}\left({ }_{2} a_{l m}+{ }_{-2} a_{l m}\right) Y_{l}^{m}(\theta, \varphi) \quad \text { and } \quad B(\theta, \varphi)=\frac{\mathrm{i}}{2} \sum_{l m}\left({ }_{2} a_{l m}-{ }_{-2} a_{l m}\right) Y_{l}^{m}(\theta, \varphi) .
$$

$E$ is invariant under parity change, whereas $B$ changes signs.

\section{References}

[1] R. D. Blandford, A. B. Saust, T. G. Brainerd, and J. V. Villumsen. The distortion of distant galaxy images by large-scale structure. Mon. Not. R. Astr. Soc. , 251:600-627, August 1991.

[2] N. Kaiser. Weak gravitational lensing of distant galaxies. Astrophys. J. , 388:272-286, April 1992.

[3] J. Miralda-Escudé. The correlation function of galaxy ellipticities produced by gravitational lensing. Astrophys. J. , 380:1-8, October 1991.

[4] D. J. Bacon, A. R. Refregier, and R. S. Ellis. Detection of weak gravitational lensing by large-scale structure. Mon. Not. R. Astr. Soc. , 318:625-640, October 2000.

[5] N. Kaiser, G. Wilson, and G. A. Luppino. Large-Scale Cosmic Shear Measurements. arXiv:astroph/0003338, March 2000.

[6] L. Van Waerbeke, Y. Mellier, T. Erben, J. C. Cuillandre, F. Bernardeau, R. Maoli, E. Bertin, H. J. Mc Cracken, O. Le Fèvre, B. Fort, M. Dantel-Fort, B. Jain, and P. Schneider. Detection of correlated galaxy ellipticities from CFHT data: first evidence for gravitational lensing by large-scale structures. Astr. \& Astrophys. , 358:30-44, June 2000.

[7] D. M. Wittman, J. A. Tyson, D. Kirkman, I. Dell'Antonio, and G. Bernstein. Detection of weak gravitational lensing distortions of distant galaxies by cosmic dark matter at large scales. Nature, 405:143-148, May 2000.

[8] H. Hoekstra and B. Jain. Weak Gravitational Lensing and Its Cosmological Applications. Annual Review of Nuclear and Particle Science, 58:99-123, November 2008. 
[9] Y. Mellier. Probing the Universe with Weak Lensing. Annual Review of Astr. 85 Astrophys. , 37:127-189, 1999.

[10] D. Munshi, P. Valageas, L. van Waerbeke, and A. Heavens. Cosmology with weak lensing surveys. Phys. Rep. , 462:67-121, June 2008.

[11] L. Fu, E. Semboloni, H. Hoekstra, M. Kilbinger, L. van Waerbeke, I. Tereno, Y. Mellier, C. Heymans, J. Coupon, K. Benabed, J. Benjamin, E. Bertin, O. Doré, M. J. Hudson, O. Ilbert, R. Maoli, C. Marmo, H. J. McCracken, and B. Ménard. Very weak lensing in the CFHTLS wide: cosmology from cosmic shear in the linear regime. Astr. ES Astrophys. , 479:9-25, February 2008 .

[12] J. Yoo, A. L. Fitzpatrick, and M. Zaldarriaga. New Perspective on Galaxy Clustering as a Cosmological Probe: General Relativistic Effects. Phys. Rev., D80:083514, July 2009.

[13] Nicola Bartolo, Sabino Matarrese, and Antonio Riotto. The Full Second-Order Radiation Transfer Function for Large-Scale CMB Anisotropies. JCAP, 0605:010, 2006.

[14] Lotfi Boubekeur, Paolo Creminelli, Jorge Norena, and Filippo Vernizzi. Action approach to cosmological perturbations: the 2nd order metric in matter dominance. JCAP, 0808:028, 2008.

[15] A. Liam Fitzpatrick, Leonardo Senatore, and Matias Zaldarriaga. Contributions to the Dark Matter 3-Pt Function from the Radiation Era. arXiv:0902.2814, 2009.

[16] Ted Pyne and Sean M. Carroll. Higher-Order Gravitational Perturbations of the Cosmic Microwave Background. Phys. Rev., D53:2920-2929, 1996.

[17] Nicola Bartolo, Sabino Matarrese, and Antonio Riotto. Gauge-invariant temperature anisotropies and primordial non-Gaussianity. Phys. Rev. Lett., 93:231301, 2004.

[18] L. Boubekeur, P. Creminelli, G. D’Amico, J. Noreña, and F. Vernizzi. Sachs-Wolfe at second order: the CMB bispectrum on large angular scales. Journal of Cosmology and Astro-Particle Physics, 08:029, August 2009.

[19] N. Bartolo, S. Matarrese, and A. Riotto. Cosmic microwave background anisotropies at second order: I. Journal of Cosmology and Astro-Particle Physics, 06:024, June 2006.

[20] C. Pitrou. The radiative transfer at second order: a full treatment of the Boltzmann equation with polarization. Classical and Quantum Gravity, 26:065006, March 2009.

[21] F. Bernardeau, L. van Waerbeke, and Y. Mellier. Weak lensing statistics as a probe of \{OMEGA\} and power spectrum. Astr. \& Astrophys. , 322:1-18, June 1997. 
[22] Asantha Cooray and Wayne Hu. Second Order Corrections to Weak Lensing by Large-Scale Structure. Astrophys. J., 574:19, 2002.

[23] M. Takada and B. Jain. Three-point correlations in weak lensing surveys: model predictions and applications. Mon. Not. R. Astr. Soc., 344:857-886, September 2003.

[24] Scott Dodelson, Edward W. Kolb, S. Matarrese, A. Riotto, and P. Zhang. Second Order Geodesic Corrections to Cosmic Shear. Phys. Rev., D72:103004, 2005.

[25] C. Shapiro and A. Cooray. The Born and lens lens corrections to weak gravitational lensing angular power spectra. Journal of Cosmology and Astro-Particle Physics, 03:007, March 2006.

[26] S. Hilbert, J. Hartlap, S. D. M. White, and P. Schneider. Ray-tracing through the Millennium Simulation: Born corrections and lens-lens coupling in cosmic shear and galaxy-galaxy lensing. arXiv:0809.5035, 2008.

[27] Elisabeth Krause and Christopher M. Hirata. Weak lensing power spectra for precision cosmology: Multiple-deflection, reduced shear and lensing bias corrections. arXiv:0910.3786, 2009.

[28] Scott Dodelson and Pengjie Zhang. The Weak Lensing Bispectrum. Phys. Rev., D72:083001, 2005.

[29] Peter Schneider, Ludovic van Waerbeke, Bhuvnesh Jain, and Guido Kruse. A new measure for cosmic shear. Mon. Not. Roy. Astron. Soc., 296:873-892, 1998.

[30] F. Bernardeau. The effects of source clustering on weak lensing statistics. Astr. \& Astrophys. , 338:375-382, October 1998.

[31] P. Schneider, L. van Waerbeke, and Y. Mellier. B-modes in cosmic shear from source redshift clustering. Astr. \& Astrophys., 389:729-741, July 2002.

[32] R. Sachs. Gravitational Waves in General Relativity. VI. The Outgoing Radiation Condition. Royal Society of London Proceedings Series A, 264:309-338, November 1961.

[33] C. Bonvin. Effect of peculiar motion in weak lensing. Phys. Rev., D78:123530, December 2008.

[34] R. M. Wald. General Relativity. The University of Chicago Press, 1984.

[35] P. Schneider, J. Ehlers, and E. Falco. Gravitational Lenses. Springer Verlag, Berlin, 1992.

[36] S. Seitz, P. Schneider, and J. Ehlers. Light propagation in arbitrary spacetimes and the gravitational lens approximation. Classical and Quantum Gravity, 11:2345, September 1994.

[37] Antony Lewis and Anthony Challinor. Weak Gravitational Lensing of the CMB. Phys. Rept., 429:1-65, 2006. 
[38] Jean-Philippe Uzan and Francis Bernardeau. Cosmic strings lens phenomenology: General properties of distortion fields. Phys. Rev., D63:023004, 2000.

[39] Matthias Bartelmann and Peter Schneider. Weak Gravitational Lensing. Phys. Rept., 340:291$472,2001$.

[40] J.N. Goldberg, Macfarlane A.J., E.T. Newman, F. Rohrlich, and E.C.G. Sudarshan. Spin-s Spherical Harmonics and $\delta$. J. Math. Phys., 8:2155-2161, 1967.

[41] Antony Lewis, Anthony Challinor, and Neil Turok. Analysis of CMB polarization on an incomplete sky. Phys. Rev., D65:023505, 2001.

[42] E.T. Newman and R. Penrose. Note on the Bondi-Metzner-Sachs Group. J. Math. Phys., 7:863-870, 1966.

[43] Irwin I. Shapiro. Fourth Test of General Relativity. Phys. Rev. Lett., 13:789-791, 1964.

[44] N. Kaiser. Nonlinear cluster lens reconstruction. Astrophys. J. Letter, 439:L1-L3, January 1995.

[45] Marco Bruni, Sabino Matarrese, Silvia Mollerach, and Sebastiano Sonego. Perturbations of spacetime: Gauge transformations and gauge invariance at second order and beyond. Class. Quant. Grav., 14:2585-2606, 1997.

[46] Anthony Challinor and Gayoung Chon. Geometry of weak lensing of CMB polarization. Phys. Rev., D66:127301, 2002.

[47] F. Bernardeau, Y. Mellier, and L. van Waerbeke. Detection of non-Gaussian signatures in the VIRMOS-DESCART lensing survey. Astr. \& Astrophys. , 389:L28-L32, July 2002.

[48] F. Bernardeau, L. van Waerbeke, and Y. Mellier. Patterns in the weak shear 3-point correlation function. Astr. \& Astrophys., 397:405-414, January 2003.

[49] Scott Dodelson, Eduardo Rozo, and Albert Stebbins. Primordial gravity waves and weak lensing. Phys. Rev. Lett., 91:021301, 2003.

[50] Devdeep Sarkar, Paolo Serra, Asantha Cooray, Kiyotomo Ichiki, and Daniel Baumann. Cosmic Shear from Scalar-Induced Gravitational Waves. Phys. Rev., D77:103515, 2008.

[51] M. Zaldarriaga and U. Seljak. All-sky analysis of polarization in the microwave background. Phys. Rev., D55:1830, February 1997. 